\title{
Common synaptic phenotypes arising from diverse mutations in the human NMDA receptor subunit GluN2A
}

\author{
Elmasri, M. ${ }^{1}$, Hunter, D. ${ }^{1}$, Winchester, G. ${ }^{1}$, Aziz, W..${ }^{1}$, Bates, E. ${ }^{1}$, Moolenaar Van Der Does, D. ${ }^{1}$, \\ Karachaliou, E. ${ }^{1}$, Sakimura, K. ${ }^{2}$ and Penn, A.C. ${ }^{1{ }^{*}}$ \\ 1. Sussex Neuroscience, School of Life Sciences, University of Sussex, Brighton, BN1 9QG, United \\ Kingdom. \\ 2. Department of Cellular Neurobiology, Brain Research Institute, Niigata University, Niigata, 951- \\ 8585, Japan. \\ * Corresponding author
}

Dominant mutations in the human gene GRIN2A, encoding NMDA receptor (NMDAR) subunit GluN2A, make a significant and growing contribution to the catalogue of published single-gene epilepsies. Understanding the disease mechanism in these epilepsy patients is complicated by the surprising diversity of effects that the mutations have on NMDARs. We have examined the cellautonomous impact of 5 severe GluN2A mutations by measuring NMDAR-mediated synaptic currents (NMDAR-EPSCs) in CA1 pyramidal neurons following rescue with human GluN2A mutants. Surprisingly, prolonged NMDAR-EPSC current decay and smaller peak amplitudes were common features of both gain- and loss-of-function mutants despite there being drastic differences between their effects on receptor function and enrichment at synapses. Modelling of NMDARs with mutant properties in CA1 neurons indicates that mutant NMDARs may contribute to broadening of depolarizations during bursts of high-frequency synaptic activity. Overall, the implication is that similar therapeutic approaches may be more widely applicable to patients with GRIN2A-related disorders irrespective of their molecular defect. 


\section{Introduction}

$N$-methyl-D-aspartate receptors (NMDARs) are conserved synaptic proteins underling the late component of postsynaptic potentials at excitatory synapses ${ }^{1}$. The receptor plays key roles in sculpting nervous system development ${ }^{2-4}$ and spine morphology ${ }^{5}$, shaping temporal integration of synaptic potentials ${ }^{6-8}$, regulating synaptic efficacy ${ }^{9-11}$, and the formation of new declarative memories ${ }^{12}$. Core to NMDARs achieving these roles is their high-calcium permeability, depolarization-dependent unblock of extracellular magnesium ions from the channel pore, and the slow time course of NMDAR channel closure (deactivation) following brief exposure to neurotransmitter glutamate ${ }^{1,10,13}$. These properties are determined to a large extent by subunit composition and stoichiometry of NMDARs ${ }^{14}$. Conventional NMDARs are heterotetramers composed of obligatory GluN1 subunits and regulatory GluN2 subunits. The GluN2 subunits contain the ligand-binding site indispensable for the glutamate-gated operation of NMDARs at excitatory synapses $^{15}$. Remarkably, four GluN2 subunits, suffixed by A-D, account for much of the diversity observed for a wide range of functional characteristics of NMDARs ${ }^{10,16,17}$.

NMDAR subunit expression varies between neuronal types and during development thus providing of means of adapting the NMDAR-mediated component of excitatory postsynaptic potentials (EPSPS) to the physiological context. Principal neurons account for the majority of neurons in the forebrain, where they predominantly express GluN2A and $2 B$, and to a much lesser extent GluN2D ${ }^{18,19}$. While GluN2B predominates at birth and continues to make a major contribution to synaptic NMDARs into adulthood, GluN2A is upregulated during early postnatal development ${ }^{18,20-}$ ${ }^{23}$, by neuronal activity ${ }^{24-26}$ and sensory experience ${ }^{27-30}$, and accounts for much of the observed developmental speeding of NMDAR-mediated excitatory postsynaptic currents (NMDAR-EPSCS) ${ }^{20,21}$. Mice with germline genetic knockout of the GluN2A gene (Grin2a) are incapable of undergoing the subunit switch and have impaired spatial learning ${ }^{31}$, altered vocalizations and spontaneous epileptiform discharges ${ }^{32,33}$. Their learning defects are also characterised by raised thresholds for the induction of and contextual fear learning and long term synaptic potentiation ${ }^{34}$,.

The significance of NMDARs is also well-demonstrated by the range of clinical phenotypes of patients with mutations in the genes encoding NMDAR subunits. Since the development of next generation sequencing methods in the mid-1990's there has been a steady rise in number of epilepsy-associated genes ${ }^{35}$ with the addition of the GluN2A receptor gene, GRIN2A, to this list only 
a decade ago ${ }^{36-38}$. Published figures now show that $>250$ patients are known to have GRIN2A mutations, either de novo or transmitted with dominant inheritance ${ }^{39,40}$. Epilepsy occurs in about $90 \%$ of these patients ${ }^{40,41}$, with GRIN2A mutations representing the best known genetic cause of Epilepsy-aphasia spectrum (EAS) disorders, where they account for $9-20 \%$ of $\operatorname{cases}^{39,42-46}$. The clinical phenotype of EAS varies considerably from the more common and benign childhood (Rolandic) epilepsy with centrotemporal spikes (CECTS), to the rarer and more severe LandauKleffner syndrome (LKS) and continuous spike-and-wave during slow wave sleep syndrome $(\text { CSWSS })^{39,47}$. Although rare, the severe epilepsy cases are often also accompanied by progressive cerebral dysfunction (epileptic encephalopathy) and are frequently comorbid with intellectual disability and language disorders ${ }^{40}$ (Figure 1B). The response to existing treatments in patients with severe EAS can be unpredictable and no one anti-epileptic drug (AED) has been demonstrated to be effective specifically in GRIN2A-related disorder ${ }^{39}$.

Most research on GRIN2A mutations has investigated the effects of missense mutations on GluN2A protein function in heterologous expression systems ${ }^{42,44,48-55}$. A common outcome of these studies is that epilepsy-associated GRIN2A mutations have strikingly variable functional consequences on NMDARs ${ }^{48,49,54,56}$. Until very recently, the impact of GluN2A mutations on the NMDAR-mediated component of synaptic transmission has received relatively little attention. While modelling of synaptic charge transfer has been made for some GRIN2A mutations ${ }^{54}$, these models do not consider how expressing the mutant GluN2A receptor could feedback on synaptic transmission ${ }^{7}$, or to what extent and how other native NMDAR subunits continue to support NMDAR-mediated synaptic transmission ${ }^{20}$. Indeed, an untested hypothesis is that GluN2B could compensate for lossof-function mutants of GluN2A ${ }^{40}$. Here, we measured NMDAR-mediated EPSCs in CA1 neurons expressing mutated human GluN2A variants to test whether similar disorders across patients with gain- and loss-of function mutations in GRIN2A could reflect common defects at the level of synaptic function. 


\section{Results}

\section{Prolongation of NMDAR-mediated synaptic currents by Grin2A null alleles}

Mutations in the gene GRIN2A vary considerably in their consequence on the expression of the encoded GluN2A protein. Recently published figures indicate that allele microdeletions and splice site mutations collectively account for $>30 \%$ of GRIN2A mutations in human disease patients ${ }^{40}$ (Figure $1 \mathrm{~A}$ ). A further $23 \%$ of the patients have either a nonsense or frame-shift mutation in the coding sequence, often before the final transmembrane domain (Figure 1A). Since the final transmembrane domain is necessary to form functional NMDAR channels ${ }^{57,58}$, nonsense and frameshift mutations proceeding it likely reflect null alleles, whether or not the mRNA is subject to non-sense mediated mRNA decay ${ }^{59,60}$. Therefore, putative null mutations may account for more than $60 \%$ of patients with GRIN2A-related disorders ${ }^{40}$.

To investigate the effect of null GRIN2A alleles on the NMDAR currents underlying excitatory synaptic transmission we have used a mouse line $\left(G \operatorname{rin} 2 a^{f|f|}\right)$ with the capacity for conditional deletion of the native mouse Grin $2 A$ alleles ${ }^{20}$. LoxP sites orientated as direct repeats and inserted in the introns flanking exon 10 predispose the intervening region to deletion by Cre-recombinase ${ }^{61}$. Exogenous expression of Cre excises the exon 10 sequence encoding the pore-lining region and third transmembrane domain of the ion channel. We reasoned that by engineering this Grin2a microdeletion in individual neurons: 1) we would isolate direct cell autonomous effects of GluN2A null alleles on excitatory synaptic transmission; and 2) we could reduce nuisance variability by having simultaneous recording of synaptic responses in neighbouring untransfected neurons evoked by the same stimulus.

We prepared slices of hippocampus from neonatal mice $\left(\operatorname{Grin} 2 a^{+/+}\right.$, Grin2 $2^{+/ f l}$ or Grin2 $\left.a^{f \mid f f}\right)$ and cultured them in vitro for a week before transfecting CA1 neurons with Cre recombinase fused inframe with Green Fluorescent Protein (GFP, Cre-GFP). Slices were cultured for a further 1.5 weeks (at 15-20 DIV) before performing simultaneous recordings of evoked synaptic currents from transfected and untransfected neurons (Figure 1B). Recording measurements were summarised as log-ratios and analysed by Bayesian multivariate, multilevel modelling (see methods; descriptive statistics of the raw data are tabulated in Supplementary Table 2). In the control case, WT $\left(\right.$ Grin $\left.2 a^{+/ t}\right)$ neurons transfected with Cre-GFP were similar to neighbouring untransfected neurons in their peak amplitude of NMDAR-mediated synaptic currents (NMDAR-EPSCs) ( $p=0.27$; Cohen's $d$ 
$(d)=-0.21$; Bayes Factor $(\mathrm{BF})=0.01 ;$ Figure $1 \mathrm{C}$ : left). In contrast, Grin2a $a^{-/}$neurons had NMDAREPSCs with peak amplitudes reduced by a factor of about $\sim 0.6$ compared to either untransfected neurons (Grin2 $a^{f|f|}, \mathrm{p}=2.7 \times 10^{-6} ; d=-1.10 ; \mathrm{BF}=21716$; Figure $1 \mathrm{C}$ : right) or Grin2 $a^{+/+}$neurons ( $\mathrm{p}=$ $1.1 \times 10^{-3} ; d=-0.89 ; \mathrm{BF}=13.1$; Figure 1D), while the peak amplitudes of NMDAR-EPSCs in neurons that were heterozygote for the null allele $\left(\right.$ Grin $2 a^{+/}$) resembled those of both untransfected neurons ( $\mathrm{p}=0.14, d=-0.29, \mathrm{BF}=0.17$; Figure $1 \mathrm{C}$ : middle) and Grin $2 a^{+/+}$neurons $(\mathrm{p}=0.77 ; d=-0.08$; $\mathrm{BF}=0.06$; Figure 1D).

Scaling the NMDAR-EPSCs to match the peak amplitudes demonstrated that Grin $2 a^{-/-}$cells have slower rates of rise and decay (Figure 1E). Compared to WT neurons, the 20-80\% rise time was 1.14-fold longer in Grin2 $a^{+-}$( $\mathrm{p}=0.0063 ; d=0.93 ; \mathrm{BF}=1.1$; Figure 1F: bottom) and 1.33-fold longer in Grin2 $a^{-/}$neurons ( $p=2.4 \times 10^{-6} ; d=1.99 ; \mathrm{BF}=1750$; Figure 1F: bottom), with the effect of heterozygosity being more convincing when compared to untransfected neurons $\left(p=4.5 \times 10^{-4} ; d=\right.$ 0.93; $\mathrm{BF}=15.4$; Figure 1F: top). NMDAR-EPSC decays were fit well by the sum of two exponentials, with the time constant of the fast component $\left(\tau_{\text {fast }}\right)$ measured in Grin $2 a^{-/}$neurons being $\sim 1.5$-fold slower than in neighbouring untransfected neurons and the contribution of the slow component to Grin $2 a^{-/}$NMDAR-EPSCs being larger by a factor of 2 (from 35.8\% to 68\%) (Supplementary Table $2)$. The net effect was an increase in the weighted decay time constant $\left(\tau_{w}\right)$ by 1.95 -fold compared to $\mathrm{WT}\left(\mathrm{p}=3.4 \times 10^{-5} ; d=3.17 ; \mathrm{BF}=1.97 \times 10^{+7}\right.$; Figure $1 \mathrm{G}$ : bottom). An appreciable effect was also observed for a single null allele, with an increase in $\tau_{w}$ by about 1.22-fold compared to either WT ( $p$ $\left.=4.7 \times 10^{-3} ; d=0.99 ; \mathrm{BF}=2.31\right)$ or untransfected neurons $\left(\mathrm{p}=2.4 \times 10^{-4} ; d=0.91 ; \mathrm{BF}=36.4\right)$ (Figure $1 G)$. The combined result of the contrasting changes in peak amplitude and kinetics of NMDAREPSCs was no discernible change in charge transfer compared to WT for either heterozygotes $(p=$ $0.27 ; d=0.29 ; \mathrm{BF}=0.10)$ or homozygotes $(\mathrm{p}=0.40 ; d=0.22 ; \mathrm{BF}=0.08)$ (Figure $1 \mathrm{H})$. Overall, these results illustrate that even a single null allele of Grin2a is sufficient to cause defects in synaptic transmission.

\section{Contrasting gain- and loss-of-function missense mutations in GRIN2A on NMDARs}

While mutations leading to premature termination of translation typically lead to loss-of-function, missense mutations cause substitutions of amino acids and can vary considerably in their consequence on protein function and expression. More than $40 \%$ of mutations discovered in GRIN2A are missense mutations (Figure 2A), with the majority of them (approx. 60\%) being in the 
sequence encoding the ligand binding domain (LBD) or the ion channel. Considerable experimental data has been published characterizing how disease-linked mutations in GRIN2A affect the surface expression and biophysical properties of GluN2A in heterologous expression systems, namely in HEK cells and Xenopus Oocytes. We compiled data for 20 different mutations from various publications and databases ${ }^{42,44,48-55}$ (Figure 2B: blue spheres). The most comprehensive sets of measurements have been made for mutations in the LBD and its linkers to the ion channel, which are the fundamental operating units for glutamate-gated opening of the channel ${ }^{62,63}$ (Figure 2B). The measured variables graphed in Figure $2 \mathrm{C}$ reflect receptor activation (agonist potencies), channel activity (open probability and current deactivation), surface expression, or a combination of the above (current density). The effects of the mutations were up to 4 orders magnitude different from WT receptors with the effects being generally larger for glutamate potency and current density (Figure 2C). Loss-of-function effects were most frequently observed for current density (79\%), surface expression (77\%) and open probability (63\%), while receptor deactivation time was the only receptor property to be dominated by gain-of-function effects (59\%) (Figure 2C).

Although mutation effects can be easily identified for specific receptor properties (Figure 2C), overall classification of mutations can become difficult as we will illustrate with some examples. The R518H mutation was identified in a patient with CSWSS and was initially described as a gain-offunction mutation based on the longer open time of the channel in single-channel recordings ${ }^{44}$. Since then, others have characterized the mutation further and reported that the mutant is poorly expressed on the cell surface and gives negligible whole-cell currents ${ }^{53,54}$, which raises uncertainty on the overall effect of this mutation. A more complicated example is the case of $\mathrm{K} 669 \mathrm{~N}$. This mutant expresses normally and exhibits a 3-4-fold higher agonist potency and deactivation time constant compared to WT receptors ${ }^{54}$. However, the open probability of the channel is estimated to be 4-5 times lower making the overall impact of this mutation also unclear.

The examples described above motivated us to adopt factor analysis to assist in interpreting the data sets of GluN2A mutant properties (Figure 2D, E). Principle component analysis provides a means to reduce multivariate data down to fewer, more manageable variables (components). The first two components calculated from the 6 variables collectively accounted for the majority of the variance $(71+16=87 \%)$. After extraction and oblique rotation of these components, the first and second components (i.e. factors)had the highest loadings for glutamate potency $\left(1 / E_{50}\right)$ and current density respectively (Figure 2D). Consistent with previous observations that GluN2 mutants 
with low glutamate potency also tend to exhibit poor surface expression ${ }^{54}$, we found a moderate but significant positive correlation between the factors 1 and $2\left(\tau_{b}=0.49,95 \% \mathrm{Cl}: 0.11-0.71 ; \mathrm{p}=\right.$ 0.015) (Figure 2E).

The bivariate distribution of factor scores for the mutations suggests possible segregation of the mutants (Figure 2E). Mutations with similar effects were classified by k-means clustering using the factor scores. The four clusters identified constituted: 1) gain-of-function mutants for the first factor (4/20); 2) mild loss-of-function mutants for one or both factors (11/20); 3) severe loss-offunction mutants for both factors (3/20); and 4) severe loss-of-function mutants for the second factor only (2/20) (Figure 2E). While the clustering of mutations is rather arbitrary and the wider population of mutations may exist rather as a continuum, the analysis serves to summarize and broadly classify this set of mutations. Given some of the assumptions and ambiguity in estimating glutamate potency for R518H and T531M (see methods), their cluster membership may not be accurate but it is clear that these represent mutations with a severe LOF phenotype. From here on, we will refer to mutations in clusters 3 and 4 more broadly as severe LOF mutants, while mutations in cluster 1 are GOF mutants.

\section{Severe gain- and loss-of-function mutations have surprisingly similar effects on NMDAR- EPSCs}

Almost half of the mutations examined above have severe and contrasting effects on NMDAR function and/or expression. To understand how these missense mutations ultimately affect the NMDAR-mediated component of synaptic transmission we adapted the protocol in Figure $1 \mathrm{~B}$ to include co-transfection of the Cre-GFP with cDNA encoding human GluN2A (Figure 3A).

Experiments with WT GluN2A showed that rescue of the peak amplitude and decay time constant of NMDAR-EPSCS was highly sensitive to the level of GluN2A expression (Figure 3B). The plasmid concentration that effectively rescued peak amplitude and decay time of NMDAR EPSCs in GluN2A $\mathrm{KO}(3.8 \mathrm{ng} / \mathrm{uL}$, Figure $3 \mathrm{~B}, \mathrm{C}$ ) was used to assess rescue of NMDAR-EPSCs for GluN2A mutants. While the expression of WT human GluN2A was capable of rescuing NMDAR-EPSCs in Grin $2 a^{-/}$CA1 neurons when compared to untransfected neurons $(\mathrm{p}=0.15 ; d=-0.34 ; \mathrm{BF}=0.165)$, NMDAR-EPSCS were smaller for both LOF (C436R: $\mathrm{p}=8.7 \times 10^{-4} ; d=-1.11$; BF $=27.8 ;$ T531M: $\mathrm{p}=3.2 \times 10^{-3} ; d=-$ $\left.0.94 ; \mathrm{BF}=7.3 ; \mathrm{R} 518 \mathrm{H}: \mathrm{p}=2.4 \times 10^{-3} ; d=-0.98 ; \mathrm{BF}=9.3\right)$ and GOF mutants (K669N: $\mathrm{p}=2.3 \times 10^{-3} ; d$ $=-0.86 ; \mathrm{BF}=9.0 ; \mathrm{L} 812 \mathrm{M} ; \mathrm{p}=1.0 \times 10^{-4} ; d=-1.04 ; \mathrm{BF}=183.6$; Figure 3D, Supplementary Table 3). 
However, the peak amplitudes were quite variable and the effects compared to WT were harder to resolve (LOF: $\mathrm{p}=0.028 ; d=-0.67 ; \mathrm{BF}=0.74 ; \mathrm{GOF}: \mathrm{p}=0.045 ; d=-0.61 ; \mathrm{BF}=0.46$; Figure $3 \mathrm{E}$ ).

A key property of NMDARs is the voltage-dependent block of the channel pore by extracellular magnesium. One of the GOF mutants, L812M has been shown to attenuate magnesium block ${ }^{55}$ and so we measured current-voltage (I-V) relationships of the NMDAR-EPSCs for each of the mutants (Figure 4A). The overall profile of the I-V curves was fairly consistent across the mutants, except for L812M, whose I-V appeared to remain linear at more negative membrane potentials. The profile of the scaled conductance-voltage (G-V) curves help to illustrate the greater conductance of NMDAREPSCS in neurons with L812M at modest negative potentials (Figure 4B). An index of rectification, the relative conductance $G_{-40 \mathrm{mv}} / \mathrm{G}_{+20 \mathrm{mv}}$ was about 1 .5-fold higher for L812M than it was for neighbouring untransfected neurons $\left(p=3.2 \times 10^{-3} ; d=0.92 ; \mathrm{BF}=8.4\right.$; Figure $\left.4 \mathrm{D}\right)$. This was largely associated with the absence of a detectable reduction in the peak amplitude at $-40 \mathrm{mV}$, unlike the results for many of the other mutations (Figure $4 \mathrm{C}, \mathrm{E}$ ).

While examining the NMDAR-EPSCs (Figure 3D), we noticed that the mutations maybe associated with changes in the decay kinetics. Indeed, the GOF mutations have been reported previously to exhibit slower deactivation in heterologous expression systems compared to WT receptors ${ }^{54,55}$. Scaling the NMDAR-EPSCS so that they have the same amplitude serves to illustrate changes in kinetics (Figure 5A), where both the severe GOF and LOF mutations were associated with more prolonged NMDAR-EPSCs. Surprisingly, the NMDAR-EPSCS in neurons with LOF mutations had a more prolonged time course than the GOF mutations, and this was confirmed by comparing their $\tau_{w}$ with those of untransfected neurons (Figure 5B: left). Specifically, putative LOF mutants C436R, T531M and R518H consistently showed a 1.8-fold slowing of the decay compared to untransfected neurons (C436R: $\mathrm{p}=2.7 \times 10^{-5} ; d=2.00 ; \mathrm{BF}=22214 ; \mathrm{T} 531 \mathrm{M}: \mathrm{p}=2.0 \times 10^{-5} ; d=2.07 ; \mathrm{BF}=3130$; $\mathrm{R} 518 \mathrm{H}: \mathrm{p}=5.1 \times 10^{-5} ; d=2.04 ; \mathrm{BF}=4642$; Figure 5B: left, Supplementary Table 3), whereas the effect of GOF mutants K669N and L812M was only 1.4-fold slower (K669N: $\mathrm{p}=1.4 \times 10^{-3} ; d=1.13$; $\mathrm{BF}=14.1 ; \mathrm{L} 812 \mathrm{M}: \mathrm{p}=4.2 \times 10^{-4} ; d=1.14 ; \mathrm{BF}=42.1 ;$ Figure $5 \mathrm{~B}$, Supplementary Table 3). Furthermore, the effects of the LOF and GOF mutations could be resolved in their contrasts with WT human GluN2A (LOF: $\mathrm{p}=1.1 \times 10^{-4} ; d=1.97 ; \mathrm{BF}=1228$; GOF: $\mathrm{p}=5.4 \times 10^{-3} ; d=1.06 ; \mathrm{BF}=2.8$; Figure 5B: right). 
The direction of the effects of missense mutations on the peak amplitude and decay of NMDAREPSCs is overall similar to those we observed for the genetic knockout in Figure 1. We therefore assessed whether or not there would be any effect on the charge transfer during NMDAR-EPSCs by taking the integral of the current traces (Figure 5C). While there appeared to be a slight increase in charge transfer for the LOF compared to untransfected neurons, the effect was not at all convincing (C436R: $\mathrm{p}=0.40 ; d=0.26 ; \mathrm{BF}=0.12 ; \mathrm{T} 531 \mathrm{M}: \mathrm{p}=0.12 ; d=0.44 ; \mathrm{BF}=0.27 ; \mathrm{R} 518 \mathrm{H}: \mathrm{p}=0.16 ; d=$ $0.41 ; \mathrm{BF}=0.23$; Figure $5 \mathrm{C}$ ). Furthermore, the GOF mutations were indiscernible from untransfected neurons with respect to charge transfer (K669N: $\mathrm{p}=0.74 ; d=-0.1 ; \mathrm{BF}=0.08 ; \mathrm{L812M}: \mathrm{p}=0.70 ; d=-$ $0.1 ; \mathrm{BF}=0.07$; Figure $5 \mathrm{C}$ ). Charge transfer of mutant NMDAR-EPSCs has been modelled by others as a function of effects on different properties of NMDARs measured in heterologous systems.

Strikingly, we do not see an obvious positive correlation between charge transfer measured in our rescue experiments compared to predictions of synaptic NMDAR-mediated synaptic currents based on the model of Swanger et al $(2016)^{54}$. To understand this discrepancy, we investigated the molecular mechanisms underlying the defects of each of the mutants.

\section{Common synaptic phenotypes of GIuN2A mutants arise from multiple, distinct molecular mechanisms}

To gain further mechanistic insight we turned to assessing the functional incorporation of mutant receptors. We accomplished this by trying to rescue NMDAR-EPSCs in the absence of native NMDARs using approaches similar to those illustrated in Figure $1 \mathrm{~B}$ and $3 \mathrm{~A}$. By recording from Crepositive neurons in cultured slices prepared from double-floxed (Grin $2 a^{f / f l} b^{f / f f}$ ) mice, we found that NMDAR-EPSCS were smaller by a factor of 0.13 compared to neighbouring untransfected neurons $\left(\mathrm{p}=2.2 \times 10^{-5} ; d=-4.1 ; \mathrm{BF}=6137\right.$; Figure $\left.6 \mathrm{~A}\right)$, indicating that the NMDAR-EPSCs were largely mediated by these two subunits. The further addition of a selective antagonist of GluN2C/D largely blocked the residual NMDAR-EPSCs $\left(p=7.2 \times 10^{-6} ; d=-5.3 ; \mathrm{BF}=86480\right.$; Figure $\left.6 \mathrm{~A}\right)$. Since most of the current was mediated by GluN2A/B we continued with experiments to rescue NMDAREPSCs from the GluN2A/B double knockout (DKO) neurons using mutant human GluN2A. Using the same concentration of WT GluN2A plasmid that we used in our earlier experiments (Figure 3-5), we found that NMDAR-EPSCs are mostly (albeit not completely) rescued by a factor of 0.7 compared to untransfected neurons. This is not surprising since the concentration transfected was originally titrated to rescue loss of a single NMDAR subunit gene. Therefore, only contrasts of mutants with respect to WT are described here and shown in Figure 6B. The LOF mutants were generally poor at 
rescuing NMDAR-EPSCs, with peak amplitudes relative to WT differing by a factor of $\sim 0.40$ (C436R: $\mathrm{p}=0.0012 ; d=-2.58 ; \mathrm{BF}=36.7 ; \mathrm{T} 531 \mathrm{M}: \mathrm{p}=0.0076 ; d=-2.31 ; \mathrm{BF}=6.5 ; \mathrm{R} 518 \mathrm{H}: \mathrm{p}=0.0069 ; d=-$ 2.21; $\mathrm{BF}=6.9$; Figure $6 \mathrm{~B})$. In contrast, $\mathrm{K} 669 \mathrm{~N}$ and $\mathrm{L} 812 \mathrm{M}$ showed functional synaptic incorporation closer to WT receptors (K669N: $\mathrm{p}=0.061 ; d=-1.23 ; \mathrm{BF}=0.74 ; \mathrm{L} 812 \mathrm{M}: \mathrm{p}=0.13 ; d=-0.99 ; \mathrm{BF}=0.36$; Figure 6B). Despite the decays of the NMDA-EPSCs being very similar between K669N and L812M in the Grin $2 a^{-/}$rescue experiments of Figure 5, the fold change relative to WT for $\tau_{\mathrm{w}}$ in the Grin $2 a^{-/}$ $\mathrm{b}^{-/}$background was 1.7 and $4.7\left(\mathrm{~K} 669 \mathrm{~N}: \mathrm{p}=1.5 \times 10^{-3} ; d=1.94 ; \mathrm{BF}=16.7 ; \mathrm{L} 812 \mathrm{M}: \mathrm{p}=2.2 \times 10^{-5} ; d=\right.$ $5.53 ; \mathrm{BF}=62570$; Figure $6 \mathrm{C}$ ). This observation is consistent with diheteromeric receptors of $\mathrm{L} 812 \mathrm{M}$ having deactivation kinetics that are 2-3 times slower than $\mathrm{K} 669 \mathrm{~N}^{54,55}$.

To test whether the poor functional incorporation of LOF mutants reflected an absence of the mutant NMDARs at synapses, we adopted a simple assay that takes advantage of the obligatory assembly of GluN1 subunits into NMDARs ${ }^{24}$ and the ER-retention of unassembled GluN1 in neurons ${ }^{64}$. GFP (or its pH-sensitive variant superecliptic pHlourin (SEP)) fused to the extracellular (or ER-lumenal) amino terminus of GluN1 has previously been used to investigate trafficking competence of different GluN2 variants to synapses ${ }^{24,52,65}$. Exogenous expression of SEP-GluN1 alone in cultured hippocampal neurons gave largely dendritic shaft fluorescence (Figure 6D). However, co-expression with WT GluN2A drove GluN1 into the cell membrane within dendritic spines, as evidenced by a 2-fold enrichment of SEP-GluN1 at spine heads colocalizing with a postsynaptic density marker, Homer1c-tdTomato. We rationalized that GluN2 mutations that disrupt the normal enrichment of NMDARs at synapses would also be incompetent at driving SEPGluN1 to synapses. Synaptic levels of NMDARs in cells expressing the C436R GluN2A mutant were smaller than for WT receptor by a factor of 0.56 (C436R: $p=3.6 \times 10^{-5} ; d=-1.57 ; B F=15928$ ), similar to neurons not co-transfected with any GluN2A (No GluN2A: $p=1.5 \times 10^{-5} ; d=-1.72 ; B F=4.7$ $x 10^{8}$ ) (Figure 6D). On the other hand, GluN2A T531M and L812M exhibited intermediate levels of SEP-GluN1 spine fluorescence (T531M: $\mathrm{p}=5.1 \times 10^{-4} ; d=-0.83 ; \mathrm{BF}=22.6 ; \mathrm{L} 812 \mathrm{M}: \mathrm{p}=0.012 ; d=-$ $0.75 ; \mathrm{BF}=1.2$, Figure $6 \mathrm{E}$ ), whereas $\mathrm{R} 518 \mathrm{H}$ and $\mathrm{K} 669 \mathrm{~N}$ efficiently rescued SEP-GluN1 to similar levels as the WT receptor $(\mathrm{R} 518 \mathrm{H}: \mathrm{p}=0.56 ; d=-0.14 ; \mathrm{BF}=22.6 ; \mathrm{K} 669 \mathrm{~N}: \mathrm{p}=0.48 ; d=0.19 ; \mathrm{BF}=1.2)$ (Figure 6D, E). We note that for the L812M mutant, the number of transfected cells appeared very low and there were signs that overexpressing GluN2A L812M could be neurotoxic, which may contribute downward bias to the synaptic levels observed in this experiment. 


\section{Missense mutations in NMDAR-receptors could contribute to altered synaptic excitability}

NMDARs are key regulators of synaptic strength and genetic deletion of NMDAR subunits has been shown to upregulate AMPAR receptor-mediated synaptic transmission ${ }^{20,66}$. To assess whether alterations to AMPAR-receptors could occur as a consequence of poor synaptic expression of some GluN2A mutants, we measured evoked AMPAR-receptor mediated EPSCs (AMPAR-EPSCs) in Grin2 $a^{-}$

- CA1 neurons that we rescued with mutated GluN2A subunits. Surprisingly, none of the mutants were associated with elevated AMPAR-EPSC peak amplitudes compared to neighbouring untransfected neurons (Figure 7A, Supplementary Table 3). In fact, one of the mutants, L812M, had marginally smaller AMPAR-EPSCs ( $p=0.032 ; d=-0.58 ; \mathrm{BF}=1.1)$, which was also evident in measurements of charge transfer $(p=0.029 ; d=-0.57 ; B F=0.97$; Figure $7 B)$, but the effect was less convincing in comparisons with WT (Peak: $\mathrm{p}=0.12 ; d=-0.65 ; \mathrm{BF}=0.31$; Charge: $\mathrm{p}=0.062 ; d=-$ $0.76 ; \mathrm{BF}=0.54)$. On the other hand, AMPAR/NMDAR ratios were larger for LOF mutants than in neighbouring untransfected neurons (Figure 7C)., but given the evidence presented in Figure 7A, 4E and 3D, this may reflect more a drop in the NMDAR-EPSC peak amplitude.

The results raise questions about how the changes in NMDAR-EPSCs by the mutations will ultimately affect excitability. In particular, how could smaller and slower NMDAR-EPSCs, alter excitability when the amount of charge transfer is essentially unchanged. Using computational models of a CA1 pyramidal neuron ${ }^{67}$, AMPA and NMDA receptors ${ }^{68}$ (Figure $8 \mathrm{~A}$ ), we simulated the effects of GOF and LOF mutations on NMDAR-EPSCs (Figure 8B, C) at two synapses in the apical dendrite: Synapse 1 (Syn 1) was located on an oblique dendrite, while the second synapse (Syn 2) was located on the main dendritic shaft. Despite using identical conductances, a larger input resistance at Syn 1 resulted in the local depolarization by synaptic activation to be 10 -fold larger than at Syn 2, thus lending Syn 1 more effective at activating their NMDARs. Both synapses were a similar distance from the soma, and the EPSPs were the same amplitude at the soma. This together with the relatively small changes in local membrane potential occurring at Syn 2, meant that Syn 2 served as a negative control for changes in the properties of the NMDAR conductance. Trains of synaptic activity delivered at the two synapses over a range of frequencies was associated with effects on EPSP summation that were both synapse and frequency dependent. As expected, NMDARs with properties modelled on the mutations were much more effective at modifying summation at Syn 1 (Figure 8E-F), where the manipulations to NMDARs became effective at train frequencies above $20 \mathrm{~Hz}$ (Figure $8 \mathrm{E}$ ). Within the $20-70 \mathrm{~Hz}$ range, the LOF- and GOF-like NMDARs 
gave smaller depolarizations with respect to the voltage integral (Figure 8D: top; 8E: bottom) despite the slower decays enhancing summation irrespective to the effects on EPSP amplitude (Figure 8E: top). At train frequencies above $70 \mathrm{~Hz}$, the effects reversed and integral of the depolarization became higher for mutant NMDARs (Figure 8D: bottom; 8E: bottom). While the peak depolarization during the train was still lower than for WT, the depolarizations became broader suggesting that mutant NMDARs may contribute to more sustained depolarizations triggered during high frequency bursts of synaptic input. Importantly, these results indicate that even in the absence of changes in charge transferred during synaptic activation, mutant NMDAR kinetics could still effectively modulate synaptic excitability.

\section{Discussion}

Our investigation revealed how mutations from patients with GRIN2A-related disorders result in defective synaptic transmission. The robust differences in the ability of mutant GluN2 subunits to rescue NMDAR-EPSCs in GluN2-null (Grin2 $a^{-/} b^{-/}$) neurons (Figure 6) was largely consistent with the classification of mutations, as severe GOF and LOF, based on analysis of data obtained in heterologous expression systems (Figure 2). Remarkably though, replacing only the native GluN2A with the mutant GluN2A subunits gave rise to NMDAR-EPSCs with very similar defects for LOF and GOF mutations: NMDAR-EPSCs that were both smaller in amplitude and slower in time course. In general, mutations phenocopied the GluN2A knockout (Figure 1), but the mechanism underlying the defect varied, with some mutants being associated with poor trafficking to synapses (Figure 6, 9B) while others reached the synapse but were either functionally silent (Figure 6, 9C) or contributed directly to the NMDAR-EPSCs through altered functional properties of the mutant receptor (Figure 6, 9D). In the case of the LOF mutations, the changes in the NMDAR-EPSCs likely reflect the contribution of natively expressed GluN2B (Figure 1B-H, 6A, 9B-C).

A current question in the field of GRIN2A-related disorders is whether expression or synaptic delivery of the native GluN2B subunit is specifically upregulated and compensates for GluN2A lossof-function ${ }^{40}$. The smaller NMDAR-EPSCs in neurons that are homozygous for the null GluN2A allele certainly indicates that there is not complete compensation by GluN2B (Figure 2C, 2D). However, in neurons heterozygous for a null allele, the slower, GluN2B-like time course of NMDAR-EPSCs in the absence of changes in amplitude suggest that there could be some, albeit small, compensation. The mechanism of this compensation may simply reflect a greater propensity for existing GluN2B- 
containing NMDARs to accumulate at synapses when synaptic content of GluN2A is reduced. This form of compensation would be consistent with a report that GluN2A deletion had no effect on either GluN2B total protein or the amplitude of ifenprodil-sensitive whole-cell currents ${ }^{40}$.

An area of wider significance also arising from our results concerns the debated existence of triheteromeric NMDARs within synapses ${ }^{69,70}$. We found that in a GluN2-null (Grin2 $a^{-\%} b^{-/}$) background, NMDAR-EPSCs in neurons transfected with GluN2B or GluN2A $\mathrm{A}_{\mathrm{L} 12 \mathrm{M}}$ had a decay time course that was 2.0 or 2.5 -fold slower than untransfected neurons, respectively. In contrast, compared to untransfected neurons NMDAR-EPSCs were only 1.5 -fold slower in Grin $2 a^{-/}$neurons rescued with GluN2A $\mathrm{A}_{\mathrm{L} 12 \mathrm{M}}$ (Figure 5A:bottom, 5B). These results are inconsistent with any simple mixture of GluN1/GluN2B and GluN1/GluN2A-L812M diheteromers and suggest that the effect of the L812M mutation may be attenuated upon forming triheteromers. Indeed, in heterologous expression systems the L812M mutation slows deactivation of glutamate-evoked currents by $10-$ fold in diheteromeric receptors (GluN1/2 $A_{L 812 M}$ ) but only about 2-fold for triheteromeric NMDARs (GluN1/2A/2A $\left.A_{L 812 M}\right)^{55}$. While similar experiments would need to be performed to confirm the reduced impact of $L 812 \mathrm{M}$ in GluN1/2A/2B receptors, our results provide support to the view that triheteromers constitute the majority of synaptic NMDARs.

The abundance of synaptic NMDARs varied considerably across the mutants. The LOF mutation C436R gave similar levels of synaptic enrichment of NMDARs as the control without GluN2A (Figure 6D-E). This mutation disrupts the formation of a disulfide bridge, which is likely key to the folding and stability of the GluN2A protein structure (Supplementary Figure 2: left). In contrast, the LOF R518H gave similar levels of NMDARs at synapses as wildtype GluN2A (Figure 6D-E), and the lack of functional incorporation (Figure 6B) may be explained by the mutation lowering glutamate potency (Figure 2C-E) by breaking coulombic interactions between GluN2A and the glutamate agonist (Supplementary Figure 2: right). Some mutations, like the T531M mutation, may even show a combination of these mechanisms (Figure 6A, 6D-E, Supplementary figure 2: middle).

In general, we were surprised by the overall modest effects of GluN2A mutations on synaptic NMDAR-EPSCs in our rescue experiments (Figures 3-5). Together with the association of these mutations with seizure disorders (Supplementary Table 4), this highlights just how sensitive the excitability of neurons is to the abundance and intrinsic properties of NMDARs. The actual impact of GRIN2A mutations on NMDAR-EPSCS may even be overestimated here since we performed our 
rescue experiments in Grin $2 a^{-/}$neurons, with the risk being that the effects we observed may not be truly representative of what occurs in the neurons of patients that are heterozygous for the mutations. This could be of particular concern for LOF mutations, in which the remaining wildtype GluN2A allele could compensate for the mutant allele. However, our experiment measuring NMDAR-EPSCs in neurons that were heterozygous for a Grin2A null allele addressed this concern (Figure 2) and suggests that qualitatively, our inferences throughout could hold in the heterozygote case.

The overall effects of GluN2A mutations on the properties of the NMDAR-EPSCs raised some interesting questions on how they may modify neuronal excitability. In particular, the slower time course and smaller amplitudes of the NMDAR-EPSCs in Grin $2 a^{-/-}$neurons rescued with GluN2A mutants culminated in charge transfer through the NMDAR channels being similar to wildtype. This prompted us to model the effect of the mutations on NMDAR-EPSCS and simulate the depolarizations caused by trains of synaptic activity onto CA1 neurons (Figure 8). While we acknowledge the relatively simple model used here, it does serve to illustrate that the slower time course of NMDAR-EPSCs mediated by GluN2A mutants can affect EPSP summation even when charge transfer is conserved. Understanding how NMDAR mutations affect synaptic excitability and network stability warrants further investigation and could be helpful in designing therapeutic strategies to treating GRIN2A-related disorders. 


\section{Methods}

Mutant classification. The functional consequences of 20 GRIN2A mutations were collected from publications and databases ${ }^{42,44,48-55}$. The functional properties were Glutamate $\mathrm{EC}_{50}$ and Glycine $\mathrm{EC}_{50}$ (in oocytes), and deactivation time constant, current density, open probability and relative cell surface levels (in HEK-293T cells). Potency for R518H was approximated from the fold change in potency for R518C documented in the CFERV database ${ }^{49}$. Potency for T531M was approximated by scaling the $\mathrm{R} 518 \mathrm{H}$ potency according to the $\mathrm{R} 518 \mathrm{H} / \mathrm{T} 531 \mathrm{M}$ ratio for single-channel opening frequency corrected for the differences in surface expression ${ }^{54}$. To handle other remaining instances of missing data, single imputation was achieved by linear interpolation following orthogonal regression on the rank-transformed data using principal component analysis (PCA) by the alternating least-squares algorithm in Matlab (Mathworks). The complete data set was then normalised to the wildtype (WT) GluN2A measurement for each property and transformed to the log scale. The data set had a Kaiser-Meyer-Olkin test statistic of $>0.5$ and was considered suitable for PCA. More than $85 \%$ of the variance was explained by the first two principal components, which were then extracted from the loadings matrix and subjected to oblique (Varimax) rotation. The resulting pattern matrix was used to calculate rotated component scores. The number of clusters chosen for K-means clustering of the scores was determined from the elbow in a plot of the sum-ofsquares error against the cluster number.

Molecular Biology. Mutagenesis was performed on the CDNA of human GRIN2A in the $\mathrm{pCl}-\mathrm{Neo}$ vector ( $p C M V$ GluN2A). GluN2A mutations with respect to the amino acid numbering in NP_000824.1 were: C436R, T531M, R518H, K669N, L812M. Oligonucleotide primers (Integrated DNA Technologies) were designed to incorporate single-base substitutions using the GeneArt ${ }^{\circledR}$ SiteDirected Mutagenesis Kit (Thermofisher Scientific) (Supplementary Table 1). The presence of each mutation was confirmed by DNA sequencing (Eurofins Genomics) and the whole coding sequence was also examined to ensure that no other mutations were introduced by polymerase chain reaction (PCR) errors. Plasmid DNA was amplified and purified using EZNA endo-free plasmid maxi kit (VWR International).

Animals. C57BI/6J mice and Sprague Dawley rats were purchased from Charles River Laboratories. Rodents were maintained under conventional housing conditions and a 12-12 light-dark cycle. 
Heterozygous Grin2A-flox (Grin2A $\left.A^{+/ f l}\right)$ and Grin2B-flox $\left(G \operatorname{rin} 2 B^{+/ f l}\right)$ mice were a gift from Kenji Sakimura (Niigata University, Japan). Generation of these mice has been described previously ${ }^{20,71}$. Homozygous Grin2A-flox (Grin2 $A^{f \mid f l}$ ) and Grin2B-flox $\left(G \operatorname{rin} 2 B^{f / f f}\right)$ mice were generated by mating the respective heterozygous mice and the colonies were subsequently maintained as homozygous lines taking care to avoid brother-sister matings. Double-flox $\left(G \operatorname{rin} 2 A^{f \mid f l} 2 B^{f / f l}\right)$ mice were generated by crossing the Grin2 $A^{f|f|}$ and Grin2 $B^{f l / f l}$ mouse lines. Ear biopsies taken for identification were used for genotyping by PCR. Primers used for Grin2A-flox genotyping were: 5'-

GAAATGTGGTAAAATCCAGTTAG-3' (forward), and 5'-TAGGCAGTAAACTTTCCTCATC-3' (reverse). Product sizes were 800 base pairs (bp) for the WT allele and $1100 \mathrm{bp}$ for the Grin2a-flox allele. Primers used for Grin2B-flox genotyping were: 5'-CCCCACTGTCTATAAAATAGAGG-3' (forward) and 5'-GCCACATAAGTTGGTCTCTT-3' (reverse). Product sizes were $900 \mathrm{bp}$ for the WT allele and $600 \mathrm{bp}$ for the Grin $2 b$-flox allele. Neonatal animals used for experiments were sacrificed by an appropriate Schedule 1 procedure (cervical dislocation) in accordance with the Animals Scientific Procedures Act 1986 amendment regulations 2012.

Cultures. Organotypic hippocampal slice cultures were prepared from neonatal mice. The hippocampi were dissected from mice at postnatal age 6-8 days in cold sterile filtered dissection medium composed of (in mM): $\mathrm{CaCl}_{2}(0.5), \mathrm{KCl}(2.5), \mathrm{KH}_{2} \mathrm{PO}_{4}(0.66), \mathrm{MgCl}_{2}(2), \mathrm{MgSO}_{4}(0.28), \mathrm{NaCl}$ (50), $\mathrm{Na}_{2} \mathrm{HPO}_{4}(0.85)$, glucose (25), $\mathrm{NaHCO}_{3}$ (2.7), sucrose (175) and HEPES (2) (pH 7.3, 330 mOsm). Transverse hippocampal slices $(350 \mu \mathrm{m})$ were cut using a Mcllwain tissue chopper (Abbotsbury Engineering Ltd., UK), placed on quartered membranes (FHLC01300, Millipore, UK) in Millicell cell culture inserts (PICM03050, Millipore, UK) and cultured in 6-well plates. The culture medium (1 ml per well) was composed of Minimum Essential Medium (MEM) supplemented with $15 \%$ heatinactivated horse serum (26050-088, Gibco), B27 supplement (17504044, Gibco), 25 mM HEPES, 3 $\mathrm{mM}$ L-glutamine, $1 \mathrm{mM} \mathrm{CaCl}_{2}, 1 \mathrm{mM} \mathrm{MgSO}_{4}, 0.25 \mathrm{mM}$ ascorbic acid and $5 \mathrm{~g} / \mathrm{L}$ glucose. Slice culture plates were kept in an incubator at $34^{\circ} \mathrm{C}$ with $5 \% \mathrm{CO}_{2}$ and $95 \%$ humidity. The culture medium was changed twice a week, with prewarmed slice culture medium supplemented with $10 \mu \mathrm{g} / \mathrm{ml}$ gentamicin.

Dissociated cultures of hippocampal neurons were prepared from neonatal Sprague Dawley rats. The hippocampus was dissected on ice and the meninges were removed in dissection medium composed of HBSS (14155048, ThermoFisher Scientific) supplemented with 10 mM HEPES (H0887, 
Sigma) and $100 \mathrm{U} / \mathrm{ml}$ Penicillin and Streptomycin (Pen-Strep, 15140122, ThermoFisher Scientific). The hippocampi were then triturated in $2 \mathrm{ml}$ of pre-warmed plating medium, which was composed of MEM (51200087, ThermoFisher Scientific) supplemented with $20 \mathrm{mM}$ glucose, $100 \mathrm{U} / \mathrm{ml}$ PenStrep, 1 mM sodium pyruvate (Sigma S8636), 25 mM HEPES, N2 supplement (17502048, ThermoFisher Scientific) and 10\% heat-inactivated horse serum (26050088, ThermoFisher Scientific). The homogenised tissue was then diluted with plating medium to $3.25 \mathrm{ml} \mathrm{per}$ hippocampus. $500 \mu \mathrm{l}$ of this was plated per well into 12-well plates that contained $16 \mathrm{~mm}$ diameter coverslips (10755354, ThermoFisher Scientific) that had been previously coated with $50 \mu \mathrm{g} / \mathrm{ml}$ polyD-lysine (P0899, Sigma) and 2 mg/ml laminin (L2020, Sigma). Neurons were allowed to settle and adhere for 3 hours at $37^{\circ} \mathrm{C}, 5 \% \mathrm{CO}_{2}$, after which, they were supplemented with $1.5 \mathrm{ml}$ culture medium containing Neurobasal-A (12349015, ThermoFisher Scientific), B-27 supplement (17504044, ThermoFisher Scientific), 100 U/ml Pen-Strep and GlutaMAX (35050038, ThermoFisher Scientific). Cultures were returned to $37^{\circ} \mathrm{C}, 5 \% \mathrm{CO}_{2}$ and fed twice a week with culture medium supplemented with antimitotic agents: $0.1 \mu \mathrm{M}$ uridine, $0.1 \mu \mathrm{M}$ fluorodeoxyuridine and $0.1 \mu \mathrm{M}$ cytosine arabinoside (all from Sigma).

Transfections. Neurons in organotypic slice cultures were transfected by single-cell electroporation ${ }^{72}$. At 6-8 days in vitro (DIV) CA1 pyramidal neurons in organotypic slices were transfected with plasmids expressing pCMV Cre-GFP with or without WT or mutant pCMV GluN2A CDNA (C436R, T531M, R518H, K669N, or L812M). Plasmid DNA was precipitated upon addition of 0.5 volume of PEG solution ( $30 \%$ PEG $8000\left(\mathrm{w} / \mathrm{v}\right.$ ) in $30 \mathrm{mM} \mathrm{MgCl}_{2}$ to isolate it from residual lipopolysaccharide endotoxin. The DNA precipitate was pelleted by centrifugation at 10,000 relative centrifugal force ( $\mathrm{rcf}$ ) for 15 minutes, washed with $70 \%$ ethanol and the air-dried pellet was redissolved in TE buffer (10 mM Tris-HCl, 1 mM EDTA). Within a week of transfection, pCMV Cre-GFP was diluted to $0.53 \mathrm{nM}\left(3 \mathrm{ng} / \mathrm{\mu L}\right.$ ) in intracellular solution containing (in $\mathrm{mM}$ ): $\mathrm{CH}_{3} \mathrm{SO}_{3} \mathrm{H}(135), \mathrm{KOH}$ (135), $\mathrm{NaCl}$ (4), $\mathrm{MgCl}_{2}$ (2), HEPES (10), Na-ATP (2), Na-GTP (0.3), spermine dihydrate (0.15), EGTA (0.06) and $\mathrm{CaCl}_{2}$ (0.01) (pH 7.25, 285 mOsm). In molecular replacement experiments, the solution also included $0.62 \mathrm{nM}(3.8 \mathrm{ng} / \mu \mathrm{L})$ of WT or mutant pCMV GluN2A. DNA-containing intracellular solution was centrifuged at $>10,000 \mathrm{rcf}$ for 15 minutes at $4^{\circ} \mathrm{C}$ to remove debris before using it to fill patch pipettes (8-10 M $\Omega$ ), which were pulled with a Flaming/Brown Micropipette puller from thick walled borosilicate glass capillaries (GB150F-8P, Science Products). Slices were transferred to the recording chamber on an upright microscope (BX51, Olympus) containing room temperature 
extracellular solution composed of (in $\mathrm{mM}$ ): $\mathrm{NaCl}$ (140), $\mathrm{KCl}$ (3), $\mathrm{MgCl}_{2}$ (1), $\mathrm{CaCl}_{2}$ (2), glucose (10), Na-pyruvate (1), $\mathrm{NaHCO}_{3}$ (2), HEPES (6), Na-HEPES (4) (pH 7.35, 300 mOsm). The patch pipette was positioned in the slice under visual guidance using a mechanical manipulator (PatchStar, Scientifica). CA1 pyramidal cells were approached with positive pressure (20 mbar). Upon dimple formation, the pressure was released to form a putative loose-patch seal. Immediately, a $12 \mathrm{~V}$ stimulus train was applied (100-200 Hz for $0.25-0.5 \mathrm{~s}$; pulse-width $0.25-0.5 \mathrm{~ms})$ from a stimulus isolator (IsoFlex, A.M.P.I.) triggered from ACQ4 software (v0.9.3) ${ }^{73}$ through a USB-X Series Multifunctional DAQ interface (NI USB-6341, National Instruments). Using the same patch-pipette, ten CA1 pyramidal neuron transfections were attempted in each slice. Typically, expression (judged from the fluorescent protein marker) was observed in half the neurons in which transfection was attempted.

Cultures of dissociated hippocampal neurons were transfected at 8 DIV using the calcium phosphate method ${ }^{74}$. DNA constructs cotransfected ( $\mu \mathrm{g} /$ coverslip) were pCaMKII- $\alpha$ Homer1ctdTomato (1), pCMV SEP-GluN1 (1.5) (Addgene plasmid \#23999), and pCMV GluN2A (1.5) or empty pcDNA3.1+ (1.5). Per well of a 12-well plate, a mixture of plasmid DNA and calcium chloride (50 $\mu$, $2.5 \mathrm{M}$ ) was added dropwise to $50 \mu \mathrm{l} 2 \mathrm{x}$ HEPES buffered saline (E1200, Promega) and incubated for 20 minutes at room temperature in the dark. Coverslips containing neurons were transferred to a fresh 12-well plate containing $500 \mu \mathrm{l}$ pre-warmed culture medium supplemented with $1 \mathrm{mM}$ kynurenic and followed by the addition of DNA/CaCl $2-H E P E S$ solution. After a 90-minute incubation at $37^{\circ} \mathrm{C}$ with $5 \% \mathrm{CO}_{2}$, the coverslips were transferred to a new 12 -well plate containing culture medium, which had been pre-incubated at $37^{\circ} \mathrm{C}$ and $10 \% \mathrm{CO}_{2}$. The 12 -well plate was then incubated at $37^{\circ} \mathrm{C}$ with $5 \% \mathrm{CO}_{2}$ for 20 minutes before being transferred back to the original 12-well plate (containing conditioned medium) and incubated at $37^{\circ} \mathrm{C}$ with $5 \% \mathrm{CO}_{2}$ until the day of imaging.

Electrophysiology. The electrophysiology method utilized throughout was current measurements in whole-cell patch-clamp configuration. Hippocampal slices had a cut made between CA3 region and $C A 1$ to reduce recurrent excitation during subsequent recordings of evoked synaptic transmission. Slices on their membrane were transferred to the chamber of an upright microscope (SliceScope Pro 2000, Scientifica), visualised using infrared light (780 nm) through an oblique condenser and 40x 0.8 NA objective (LUMPLFLN40XW, Olympus) and captured using a mono charge-coupled device (CCD) monochrome camera (SciCam, Scientifica) controlled using Scight 
software (Scientifica). The tissue was held down with a slice anchor and perfused with artificial cerebrospinal fluid (ACSF) solution containing (in $\mathrm{mM}$ ): $\mathrm{NaCl}(125), \mathrm{KCl}(2.5), \mathrm{NaHCO}_{3}$ (25), glucose (10), Na-pyruvate (1), $\mathrm{MgCl}_{2}$ (1) and $\mathrm{CaCl}_{2}$ (2). $5 \mu \mathrm{M}$ 2-chloroadenosine (ab120037, Abcam) was added to prevent epileptic activity. Addition of other drugs depended on the experiment (see below). The ACSF was bubbled with carbogen $\left(95 \% \mathrm{O}_{2}, 5 \% \mathrm{CO}_{2}\right)$ and maintained at $30^{\circ} \mathrm{C}$ with an inline heater (SM-4600, Scientifica) and heated chamber (HCS, ALA Scientific; TC-10, NPI Electronic). A bipolar tungsten stimulation electrode was positioned (LBM-7, Scientifica) in Stratum radiatum to stimulate the Schaffer collateral pathway. 2-4 $M \Omega$ patch pipettes for whole-cell patch clamp recordings were filled with intracellular solution composed of (in $\mathrm{mM}$ ): $\mathrm{CH}_{3} \mathrm{SO}_{3} \mathrm{H}$ (120), $\mathrm{CsOH}$ (120), $\mathrm{CsCl}(20), \mathrm{MgCl}_{2}$ (2.5), HEPES (10), Na-ATP (4), Na-GTP (0.4), phosphocreatine disodium salt (5) and EGTA (0.2) ( $\mathrm{pH} 7.25)$. Osmolarity of intracellular and extracellular solutions was checked and adjusted on the day of each experiment and adjusted by addition of water or the major salt so that the ACSF osmolarity was $10-15 \mathrm{mOsmol} / \mathrm{kg}$ greater than the intracellular solution. To account for inter-slice variability in our recordings, two CA1 neurons were patched simultaneously - one transfected neuron identified by the Cre-GFP fluorescence (pE-300 white, CoolLED; 49018 EGFP long-pass, Chroma) and one neighbouring untransfected neuron. The intensity and polarity of the constant voltage stimulus (50 $\mu$ s duration) was adjusted (IsoFlex, A.M.P.I.) to obtain reliable NMDAR responses that were $<250 \mathrm{pA}$. Stimuli were delivered at an inter-sweep interval of $10 \mathrm{~s}$. Signals were acquired with a MultiClamp 700B (Molecular Devices), low-pass filtered (4 kHz, 4-pole Bessel) and digitized ( $25 \mathrm{kHz}$ ) directly to hard disk using a USB-X Series Multifunctional DAQ interface (NI USB-6363, National Instruments). Hardware was controlled using Python-based ACQ4 software $(v 0.9 .3)^{73}$. The series resistance was left uncompensated.

To isolate NMDAR current, the ACSF included $50 \mu \mathrm{M}$ picrotoxin (ab120315, Abcam) and $10 \mu \mathrm{M}$ gabazine (SR95531, Abcam) to block ionotropic gamma-amino-butyric acid receptors (GABA $A_{A} R$ ) and $10 \mu \mathrm{M}$ NBQX (1044, Tocris Biosciences) to inhibit $\alpha$-amino-3-hydroxy-5-methyl-4isoxazolepropionic acid receptors (AMPARs). The membrane potential was raised from -75 $\mathrm{mV}$ to $+20 \mathrm{mV}$ at a rate of $0.5 \mathrm{mV}$ per second to provide relief from the magnesium block on NMDARs. For each neuron, 30 evoked NMDAR-EPSCs were recorded.

For molecular replacement experiments, mixed AMPAR- and NMDAR-EPSCs were recorded at a range of voltages $(-100,-80,-60,-40,-20,0$ and $+20 \mathrm{mV})$. These holding potentials account for the 
liquid-liquid junction potential, which we calculated to be $+10 \mathrm{mV}$ for our solutions (PowerPatch Tools Igor Pro Tool collection). The ACSF included $50 \mu \mathrm{M}$ picrotoxin, $10 \mu \mathrm{M}$ gabazine as above, and $2 \mu \mathrm{M}$ CGP52432 to inhibit GABA ${ }_{B}$ receptors. The intracellular solution was supplemented with 3 $\mathrm{mM} \mathrm{QX}-314-\mathrm{Cl}$ to inhibit voltage-activated sodium and calcium channels. For each voltage, 10 evoked NMDAR-EPSCS were recorded per neuron at an inter-sweep interval of $10 \mathrm{~s}$. Transfections and recordings for GluN2A mutant molecular replacements were randomized and performed double blind. All molecular replacement experiments were carried out in slice cultures prepared from the left hemisphere since the measurements of NMDAR-EPSC properties tended to show a lower variance compared to those in neurons of slices from the right hemisphere.

Electrophysiological recordings were analysed using a custom python module (https://github.com/acp29/penn) and Stimfit software (v0.13) ${ }^{75}$. Pairs of neurons were discarded if the difference in access resistance varied by more than $\sim 8 \mathrm{M} \Omega$. Decay of the EPSC currents was fit by a two-component exponential decay with offset initially using the Chebyshev algorithm, where the fit parameters were then used as starting values for further optimization by the LevenbergMarquardt algorithm. The weighted exponential time constant was calculated as the sum of component time constants multiplied by their fractional amplitude. Where the two-component fits failed, a single exponential was found to fit well. Approximate measures of whole-cell properties (including access resistance, whole-cell capacitance, input resistance and specific membrane resistance) were calculated from the current response to a $-5 \mathrm{mV} 20$ ms voltage step, which was included in all recording sweeps.

The mutants selected for recordings in this study are known to have no more than 2.5 -fold changes in magnesium potency ${ }^{49,55}$. We thus separated AMPAR- and NMDAR-components of evoked mixed EPSCs during the analysis using the following steps (See Supplementary Figure 1): 1) The current traces at each holding potential were converted to conductance using Ohm's law; 2) The AMPARmediated conductance at $-100 \mathrm{mV}$ was subtracted from the conductance traces at $-40 \mathrm{mV}$ and +20 $\mathrm{mV}$. The resulting conductance traces thus reflect AMPAR-EPSCs $(-100 \mathrm{mV})$ and NMDAR-EPSCs $(-40$ $\mathrm{mV}$ and $+20 \mathrm{mV}$ ). Amplitude, decay and charge were measured in both AMPAR and NMDAR conductance traces. The conductance trace at $-40 \mathrm{mV}$ reflects an NMDAR-EPSC partially attenuated by voltage (i.e. magnesium block) and was used to calculate a rectification index: peak conductance of NMDAR-EPSCS amplitudes EPSC-40mv / EPSC $+20 \mathrm{mv}$. We make the reasonable assumption in this 
analysis that the current-voltage relationship of the AMPAR-EPSC is linear and that most of the NMDAR current is blocked at the $-100 \mathrm{mV}$ holding potential.

Imaging. Transfected cultures of dissociated hippocampal neurons were imaged at DIV11. Neurons were imaged using an inverted microscope (AxioVert.A1, Zeiss), a 40x 0.55 NA objective and a complementary metal-oxide-semiconductor (CMOS) camera (ProgRes GRYPHAX Arktur 8 MPix, Jenoptik). Coverslips were placed in ASCF composed of (in $\mathrm{mM}$ ): $\mathrm{NaCl}(140), \mathrm{KCl}(3), \mathrm{MgCl}_{2}$ (4), $\mathrm{CaCl}_{2}$ (4), glucose (10), Na-pyruvate (1), HEPES (6), Na-HEPES (4) (pH 7.35, 300 mOsm). Neurons were imaged live for the GluN1-SEP signal at (LED $\lambda_{\text {ex }} 470 \mathrm{~nm}$ ) and the Homer1c-tdTomato signal at (LED $\lambda_{\text {ex }} 530 \mathrm{~nm}$ ). Five images (8-bit) were taken for each coverslip and converted to grayscale in FIJI software ${ }^{76,77}$. Red and green images were aligned if necessary with custom macros using a modified version of the Align_RGB_planes plugin available at https://github.com/acp29/pimage/tree/master/Penn/align_rgb). The background fluorescence was subtracted from both images, and puncta in the Homer1C-tdTomato signal were selected semiautomatically as regions of interest (ROIs). The ROIs were overlaid onto the GluN1-SEP signal and the mean pixel intensity was measured at these ROIs. Custom macros used for the measurement of spine fluorescence analysis are available at https://github.com/acp29/pimage/blob/master/Penn/pimage.ijm. The experimenter was blind to the experimental groups during image analysis, and the results were reproduced by multiple lab members.

\section{EPSP simulations}

Simulations were conducted in NEURON 7.5 software $^{78}$. All simulations were carried out in a CA1 pyramidal model neuron developed to accurately capture voltage attenuation ${ }^{67}$. The model includes both passive leak $\left(\mathrm{Na}^{+}\right.$and $\mathrm{K}^{+}$) and cyclic-nucleotide-gated (HCN) channels, distributed non-uniformly across the model. In all cases simulations were initiated with a time step of $25 \mu \mathrm{s}$ and at a temperature of $35^{\circ} \mathrm{C}$. Simulated synaptic inputs consisted of both AMPAR and NMDAR conductances described by the sum of two exponential ordinary differential equations. AMPAR receptor rise and decay time constants were set to $0.3 \mathrm{~ms}$ and $3 \mathrm{~ms}$ respectively and maximal conductance $\left(g_{\max }\right)$ was set to $500 \mathrm{pS}$. WT NMDAR rise and decay time constants were set to 3 ms and $80 \mathrm{~ms}$ respectively. The rise and decay time constants of NMDAR conductances were scaled by 
a factor of 1.5 or 2.0 to approximate the kinetics of GOF and LOF mutations on NMDAR-EPSCs. The NMDAR $g_{\max }$ was set to $1 \mathrm{nS}, 690 \mathrm{pS}$ and $540 \mathrm{pS}$ to emulate WT, GOF and LOF NMDARs respectively, with constant charge transfer. Synaptic inputs were positioned in one of two locations, both around $300 \mu \mathrm{m}$ from the soma. Synapse 1 (Syn 1) was on an oblique dendrite (off the main apical branch) whilst synapse 2 (Syn 2) was on the trunk of the main apical denrite. The dendritic diameter at the location of Syn 1 was $6 x$ smaller than that of Syn $2(0.4 \mu \mathrm{m}$ and $2.4 \mu \mathrm{m}$ respectively) and was associated with $\sim 10 x$ larger EPSP in response to the same input conductance. For each experimental run, barrages of synaptic events were generated in trains of 10 at frequencies ranging from 1 to $10 \mathrm{~Hz}$ in $1 \mathrm{~Hz}$ steps; 10 to $100 \mathrm{~Hz}$ in $10 \mathrm{~Hz}$ steps; and $200 \mathrm{~Hz}$. The models and experiments that were conducted were all deterministic in nature. No stochastic mechanisms, such as open probability of receptors or random assignment of parameters, were included in the model.

\section{Statistics}

The sample size in most experiments aimed to satisfy the resource equation, where the error degrees of freedom in a mixed linear model framework would reach between 10-20. Matched measurements of NMDAR-EPSC and whole-cell properties in transfected and untransfected where expressed as log-ratios and included in multivariate outlier detection by robust principal component analysis (ROBPCA) using the LIBRA Matlab library ${ }^{79,80}$ in Matlab 9.2 (R2017a, Mathworks). ROBPCA produces principal components that are representative of the trend of the majority of the data with lower influence from extreme values. The number of PCs used was the number that explained $90 \%$ of the variance. Samples that were extreme orthogonal outliers, bad leverage points and were beyond the cut-off value of $\sqrt{ } X^{2}, 0.975$ were excluded from all subsequent analysis.

Statistical inference was carried in R by fitting Bayesian generalized linear multivariate multilevel models using brms and 'Stan' ${ }^{81}$. For the electrophysiology data, fitting was performed on the $\log _{\mathrm{e}}$ transformed transfected/untransfected ratios using a model that accounted for the hierarchical data structure to avoid pseudo-replication:

$$
\log (\text { ratio }) \sim 0+\text { genotype }+(1 \mid \text { animal } / \text { slice })
$$

Where multiple response variables were measured (e.g. decay, rise), these were listed in a multivariate brms formula with rescor set to TRUE to model residual correlations. 
For the imaging data, fitting was performed on the $\log _{\mathrm{e}}$-transformed ratio of SEP-GluN1/ Homer1CtdTomato calculated from mean fluorescence intensities of the synapse ROls for each transfected neurons. The model accounted for the nesting of neurons within experimental repeats to avoid pseudo-replication:

$$
\log (\text { ratio }) \sim 0+\text { genotype }+(1 \mid \text { experiment })
$$

Markov Chain Monte Carlo simulations in brms used NUTS sampling, which does not require priors to be conjugate. Since log-ratios can be represented as proportions via the inverse logit transform, and because a standard uniform distribution is an intuitive and objective choice of prior for proportions, we used the standard logistic distribution as a prior for the population effects (i.e. of the log-ratios) in our Bayesian models. Simulations were run as 4 independent chains, each with 5000 samples warmup and 5000 samples post-warmup (20,000 total post-warmup). The Student-t distribution family was used, enabling robust linear regression that is less influenced by potential outliers. The adapt delta value was raised as necessary to ensure convergence of the chains, which was assessed by ensuring that the potential scale reduction factor on split chains (Rhat) was 1.00. Convergence was also assessed by visually inspecting plots of the post-warm up chains of the posterior values. The effective sample sizes (i.e. independent information in autocorrelated chains) were typically $>10,000$. R packages emmeans and tidybayes were used to calculate equi-tailed (central) 95\% credible intervals of the posterior distributions. Posterior distributions and credible intervals are all presented graphically after back-transformation to the ratio scale because this is more intuitive to read and interpret. Hypothesis test results are presented in the text as, a twosided p-value, a Cohen's $d$ standardized effect size (of the log-ratio) and a Bayes Factor (BF). Inference was based on the weight of evidence considering all these statistics rather than binary decisions using significance level thresholds alone. Frequentist-like p-values and Bayes Factors (with a point null) were calculated using the bayestest $R$ package ${ }^{82}$, where Bayes factors used the same logistic distribution prior on log-ratios that was used for model building. Orthonormal contrasts were used throughout to ensure that the marginal prior on all effects was identical. P-values are summarised on the graphs where: $*=p<0.05 ; * *=p<0.01 ; * * *=p<0.001 ; * * * *=p<0.0001$; no annotation corresponds to $p>0.05$. Within the Bayesian framework of multilevel model building, use of the same prior across the parameters results in partial pooling, which efficiently addresses concerns of multiple comparisons ${ }^{83}$. 
Confidence intervals and p-values for Kendall's tau-b $\left(\tau_{b}\right)$ (non-parametric) correlation coefficients were calculated using the iboot toolbox (v2.8.7.3, https://github.com/acp29/iboot) run in Matlab. Central coverage of bias-corrected and accelerated ( $\mathrm{BCa}$ ) intervals was calibrated to reduce small sample bias using a double bootstrap procedure, with 20,000 and 200 resamples for the first and second bootstrap respectively. 


\section{Acknowledgements}

We gratefully acknowledge the following kind gifts: pCMV Cre-GFP from Christophe Mulle (IINs, Bordeaux, France), pCMV GluN2A from Stephen Traynelis and Hongjie Yuan (Emory, Atlanta, Georgia, USA) and pCaMKII- $\alpha$ Homer1c-tdTomato from (IINs, Bordeaux, France). The PhD studentship of M.E. was funded on a University of Sussex Life Sciences School studentship. A.C.P. was funded on MRC Career Development Award (MR/M020746/1), in which W.A. and M.E. were funded as Post-docs. 


\section{Figures}

Figure 1
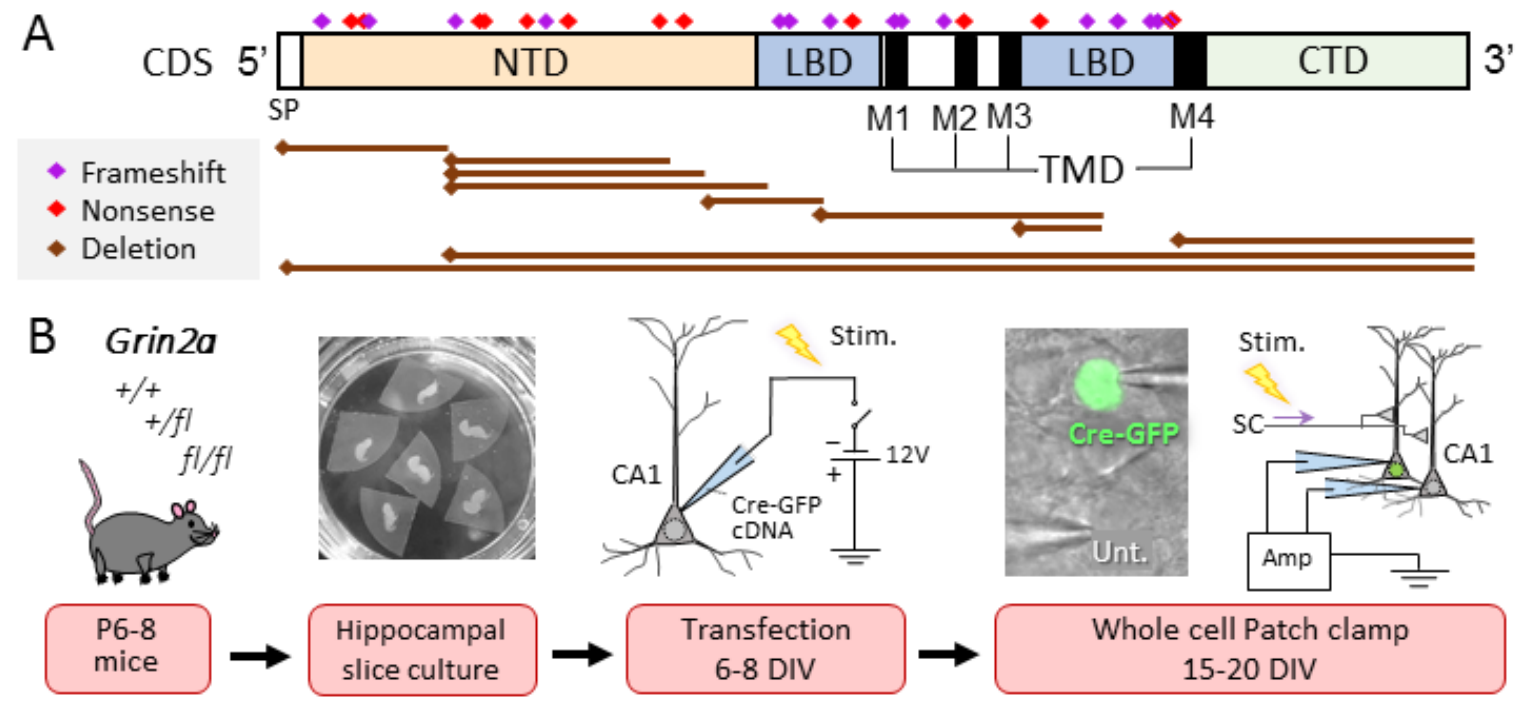

C
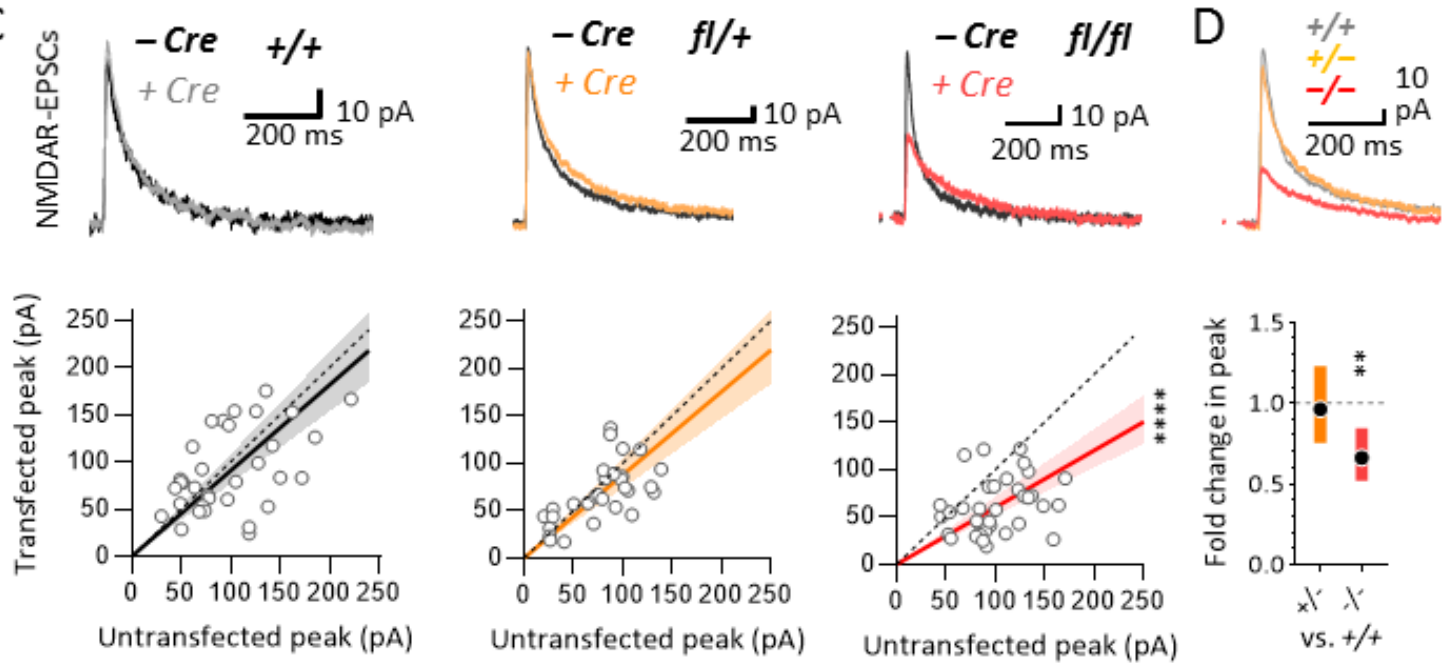

$\mathrm{E}$

Untransfected peak (pA)

Untransfected peak (pA)

Untransfected peak (pA)

$\mathrm{F}$

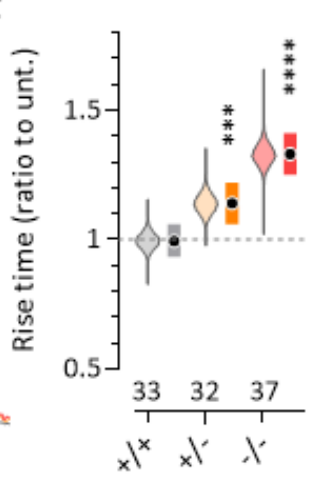

$2 \mathrm{~ms}$
G
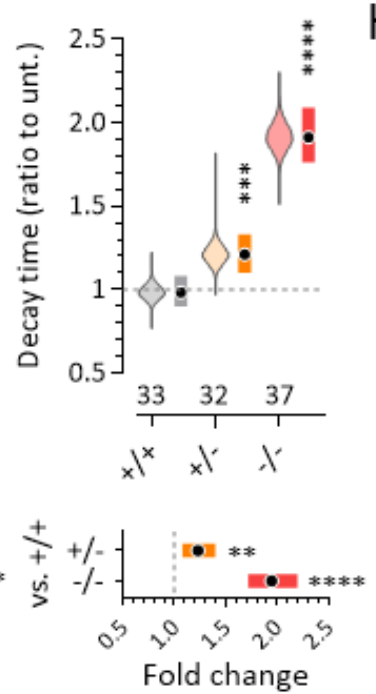

Fold change
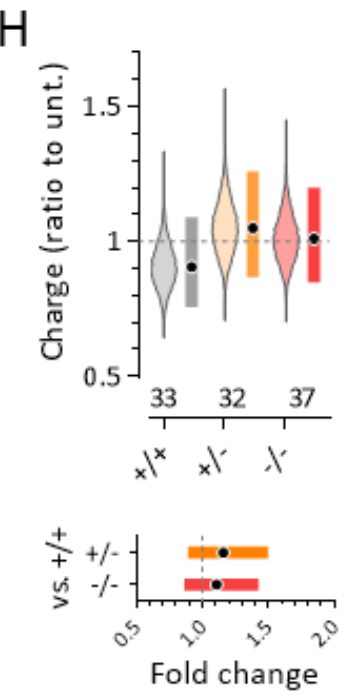


\section{Figure 1: Dose-dependent effects of GluN2A on NMDAR-EPSC kinetics in a cell- autonomous model of GRIN2A null alleles.}

A. Abundance and location of different types of null GRIN2A mutations classified as pathogenic according to the guidelines by the American College of Medical Genetics and Genomics (ACMG) ${ }^{40}$. Pathogenic protein truncating mutations largely occur prior to the $4^{\text {th }}$ transmembrane domain (M4). Abbreviations: CDS = coding sequence; NTD = amino terminal domain; LBD = ligand-binding domain; TMD = transmembrane domains; $C T D$ = carboxy-terminal domain.

B. Illustration of the experiment used to test the cell autonomous effect of heterozygosity or homozygosity for a null Grin2a allele on NMDAR mediated EPSCS in CA1 pyramidal neurons. C. Top: Representative traces of NMDAR-EPSCs from transfected and untransfected cell pairs. Bottom: Scatter plots of NMDAR-EPSC peak amplitudes measured for pairs of transfected and untransfected neurons. Also graphed is the line of unity (dotted), median (solid) and 95\% credible interval ( $\mathrm{Cl}$ ) for the posterior distributions of the transfected/untransfected ratio (shaded).

D. Top: Representative NMDAR-EPSC traces from neurons of each genotype overlaid. Bottom: Median and $95 \% \mathrm{Cl}$ of the posterior distributions for the contrasts between the data shown in C versus $+/+$ control.

E. Left: Peak-scaled, representative NMDAR-EPSCs traces from neurons of each genotype are overlaid and presented on different time scales to illustrate the effects of genotype on the rise and decay time course.

F. Violin plots of the posterior distributions along with their median and $95 \% \mathrm{Cls}$ for the transfected/untransfected ratios of the NMDAR-EPSC $20-80 \%$ rise time (top) and their contrasts versus $+/+$ control (bottom).

G. As in $\mathrm{F}$, but for the weighted decay time constant.

H. As in $F$, but for the charge transfer.

C-H. NMDAR-EPSCs were recorded at $+20 \mathrm{mV}$ in the presence of AMPAR receptor antagonist NBQX $(10 \mu \mathrm{M})$ (see methods). Posterior distributions of the log-ratios were simulated using Bayesian multivariate multilevel modelling with a standard logistic distribution for a prior (see methods). The number of neuron pairs is provided at the base of each graph. 


\section{Figure 2}

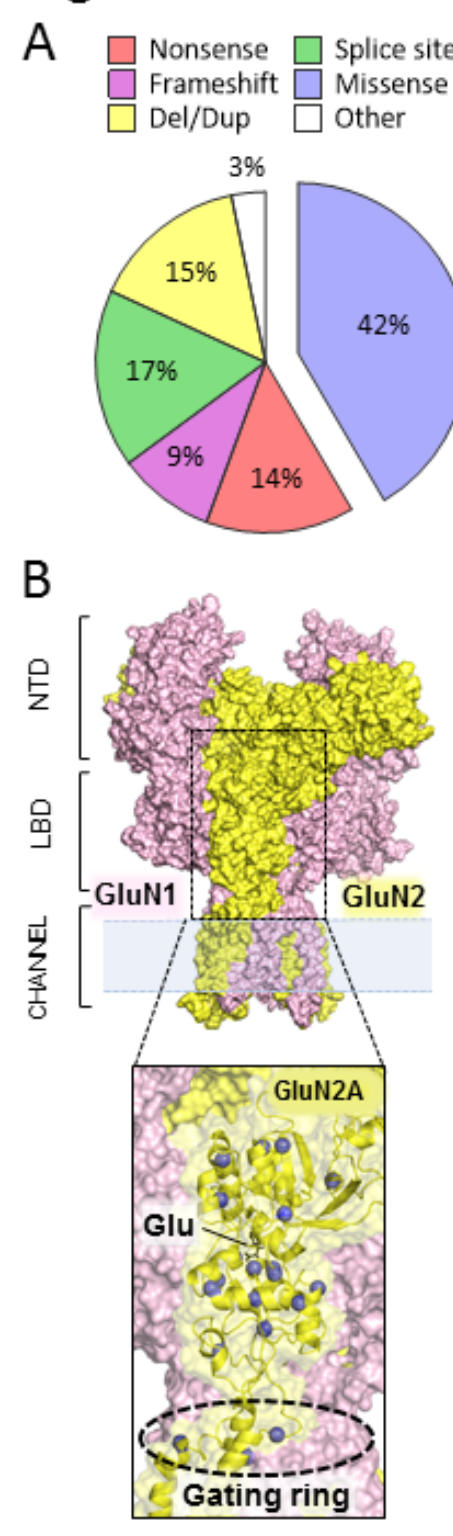

C
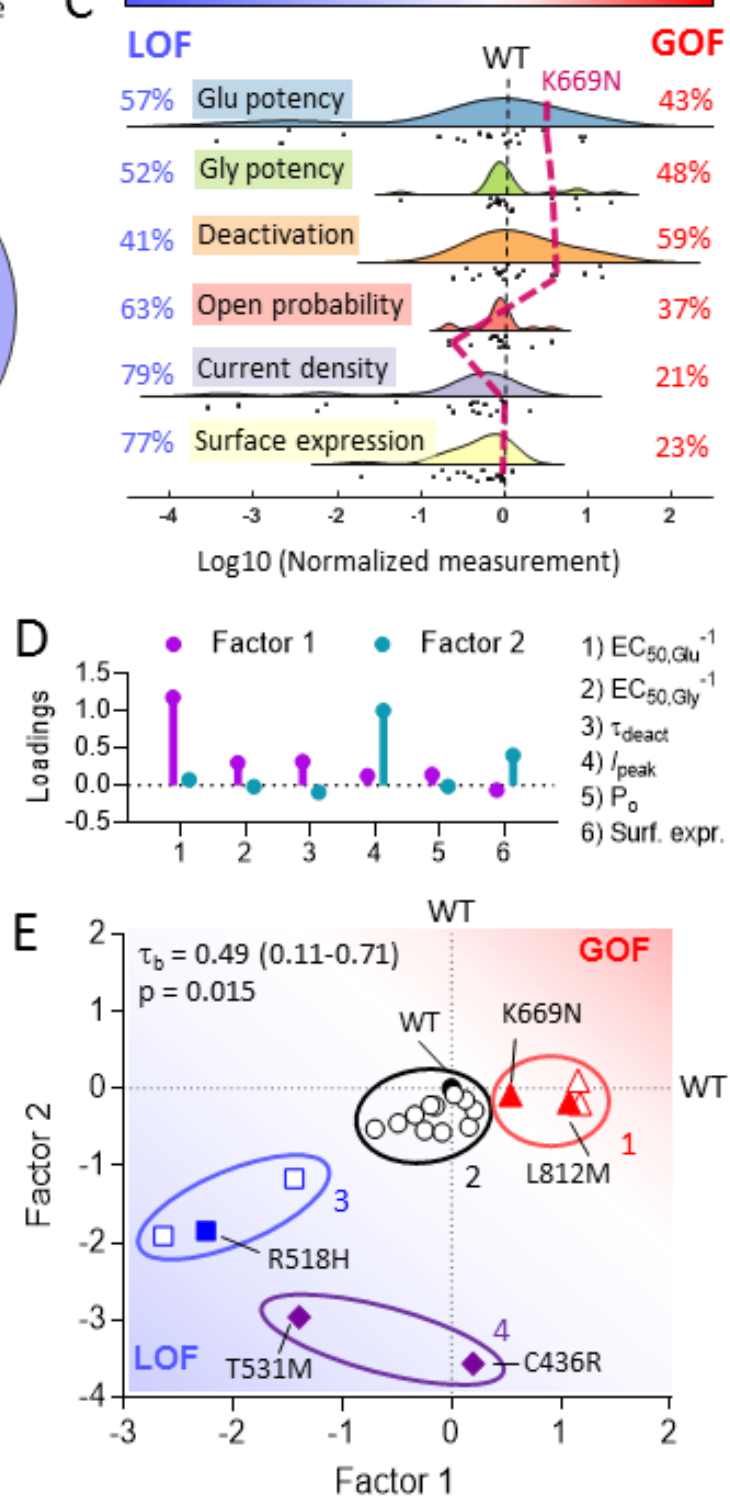

\section{Figure 2: Missense GRIN2A mutations can impart severe overall gain or loss of function effects on NMDARs.}

A. A pie chart illustrating the proportion of different types of mutations in human GRIN2A. Missense mutations make up just over $40 \%$ of all GRIN2A mutations but their impact on NMDAR function is harder to predict.

B. Surface representation of an NMDAR heterotetramer of GluN1 and GluN2 (pink and yellow respectively). The ligand-binding domain (LBD) of GluN2A is boxed out and magnified to illustrate the glutamate (Glu) binding site, the adjacent gating ring and the location of some functionally 
characterized disease-associated missense mutations (blue spheres) ${ }^{42,44,48-55}$. NTD $=$ Amino terminal domain.

C. Raincloud plot showing quantification of the gain $(>0)$ and loss $(<0)$ of function of the mutations shown in $B$ with respect to different properties measured for mutant GluN1/2A receptors expressed in HEK cells or Oocytes ${ }^{42,44,48-55}$. The black dashed line at 0 corresponds to WT GluN2A. The tick dashed maroon line corresponds to the $\mathrm{K} 669 \mathrm{~N}$ mutation, which serves to illustrate that some mutations can be simultaneously be gain-of-function, loss-of-function or have no effect across different properties of the receptor.

D. Leaf and stem plot of the loadings (pattern matrix) of the first two components of a principle component analysis after oblique rotation. The loading for first factor is highest for glutamate potency $\left(\mathrm{EC}_{50, \mathrm{Glu}}{ }^{-1}\right)$, while the loading for the second factor is highest for peak current density $\left(I_{\text {peak }}\right)$. E. Scatter plot of the scores of the 20 GRIN2A mutations (shown 2B-C) for each of the factors 1 and 2 from $D$. The graph is annotated with the results of K-means clustering: 1 - GOF mutants (red); 2 mutants with mild LOF or GOF (black); 3 - mutants with severe LOF for both factors (blue); 4 mutants with severe LOF for factor 2 only (purple). Filled symbols represent mutations used for experiments in this study. The graph is annotated with Kendall's tau-b correlation coefficient accompanied by bootstrap confidence intervals and $\mathrm{p}$-values (see methods). 


\section{Figure 3}
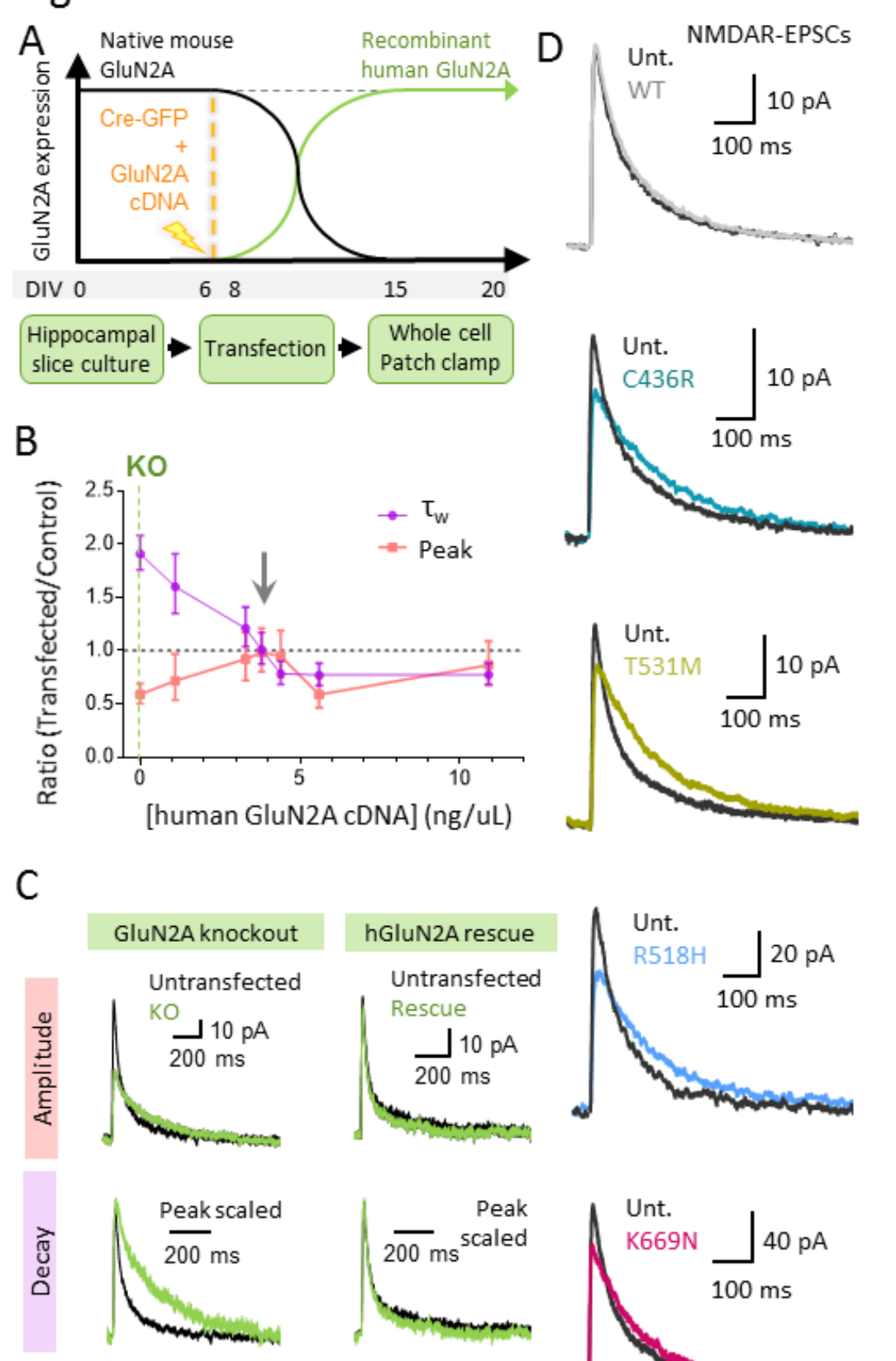

E
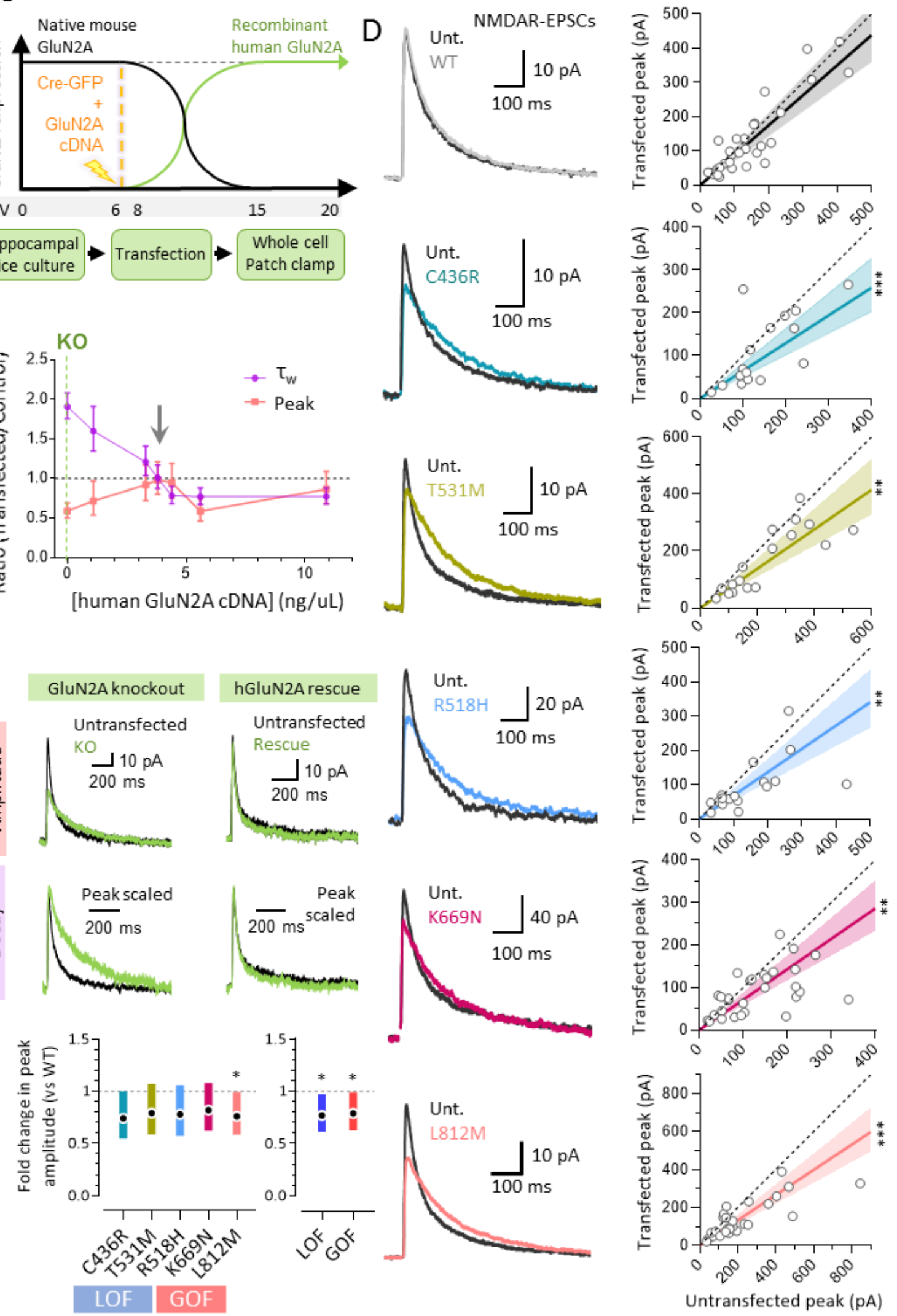

Untransfected peak (pA) 


\title{
Figure 3: Both gain- and loss-of-function LBD mutants are less effective at rescuing NMDAR-mediated EPSCs compared to WT GIuN2A
}

\author{
A. The experimental approach illustrated in Figure 1B was adapted to investigate the effect of \\ missense mutations, by co-expressing Cre-GFP with human GluN2A. After transfection, the action of \\ Cre results in the decline of native mouse GluN2A expression while it is replaced by the co- \\ expressed human GluN2A cDNA.
}

B. The transfected/untransfected ratios for NMDAR-EPSC peak amplitude and weighted decay time constants for varying amounts of co-transfected human GluN2A cDNA. Arrow indicates the concentration that effectively rescued the peak amplitude and decay time constant of NMDAREPSCS. Data shown are the median and $95 \% \mathrm{Cl}$ of the posterior distributions.

C. Representative traces of NMDAR-EPSCS recorded from an untransfected neuron and a neuron that is homozygous KO for Grin2a (fl/fl), or its rescue with human GluN2A.

D. Left: Representative traces of NMDAR-EPSCs from transfected and untransfected cell pairs. Transfected neurons represent rescue with human GluN2A (WT or mutant). Right: Scatter plots of NMDAR-EPSC peak amplitudes measured for pairs of transfected and untransfected neurons. Also graphed is the line of unity (dotted), median (solid) and 95\% credible interval (Cl) for the posterior distributions of the transfected/untransfected ratio (shaded).

E. Median and $95 \% \mathrm{Cl}$ of the posterior distributions for the contrasts between the data shown in $\mathrm{D}$ versus WT control.

B-E. NMDAR-EPSC conductances were calculated from current recordings at $+20 \mathrm{mV}$ by subtracting the AMPAR-EPSC conductance recorded at $-100 \mathrm{mV}$ (see methods). NMDAR conductances where converted back to currents for the figures and scatter plots.

B, D and E. Posterior distributions of the log-ratios were simulated using Bayesian multivariate multilevel modelling with a standard logistic distribution for a prior (see methods). 


\section{Figure 4}
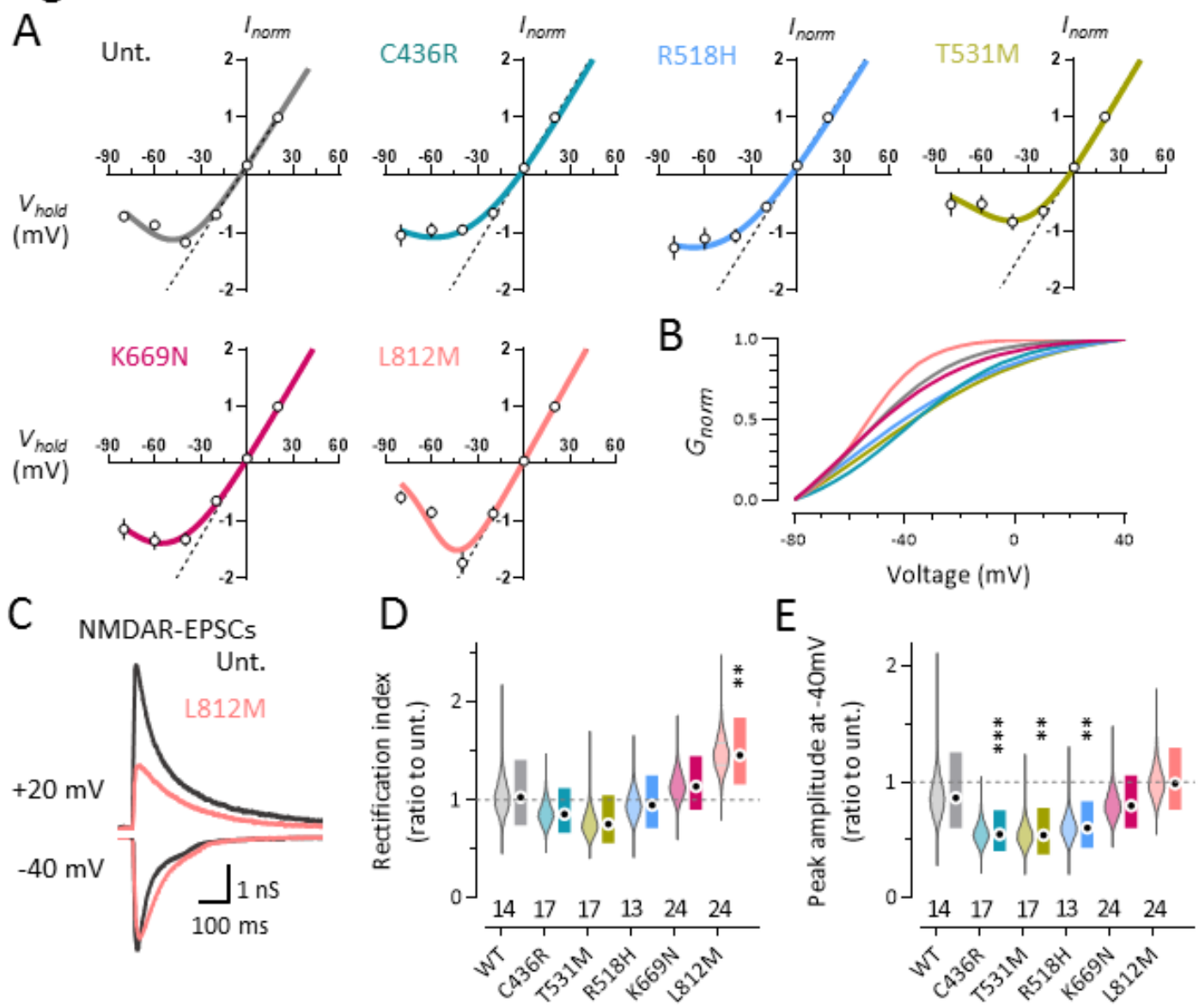

\section{Figure 4: Varied effects of GluN2A mutants on current-voltage relationships of NMDAR-mediated EPSCs}

A. Current-voltage (I-V) relationships of NMDAR-EPSCS for untransfected neurons, or neurons rescued with human GluN2A mutants. The bold line represents a fit to the data using the Boltzmann equation (see methods).

B. Full scale normalization of conductance-voltage (G-V) curves calculated from the fits to I-V curves in A. NMDAR-EPSCs for the mutant L812M appear to have greater voltage dependence than the other mutations.

C. Example NMDAR-EPSC traces at $+20 \mathrm{mV}$ and $-40 \mathrm{mV}$ for an L812M-rescue and untransfected neuron.

D. Quantification of G-V curves by a rectification index $\left(G_{+20 m v} / G_{-40 m v}\right)$. Violin plots of the posterior distributions along with their median and $95 \% \mathrm{Cls}$ for the transfected/untransfected ratios of the NMDAR-EPSC rectification index. 
E. Violin plots of the posterior distributions along with their median and $95 \% \mathrm{Cls}$ for the transfected/untransfected ratios of the NMDAR-EPSC conductance at $-40 \mathrm{mV}$.

A-E. NMDAR-EPSC conductances were calculated from current recordings at holding potentials between -80 and $+20 \mathrm{mV}$ by subtracting the AMPAR-EPSC conductance recorded at $-100 \mathrm{mV}$ (see methods). NMDAR conductances where converted back to currents for the I-V curves.

D and E. Posterior distributions of the log-ratios were simulated using Bayesian multivariate multilevel modelling with a standard logistic distribution for a prior (see methods). The number of neuron pairs is provided at the base of each graph. 


\section{Figure 5}
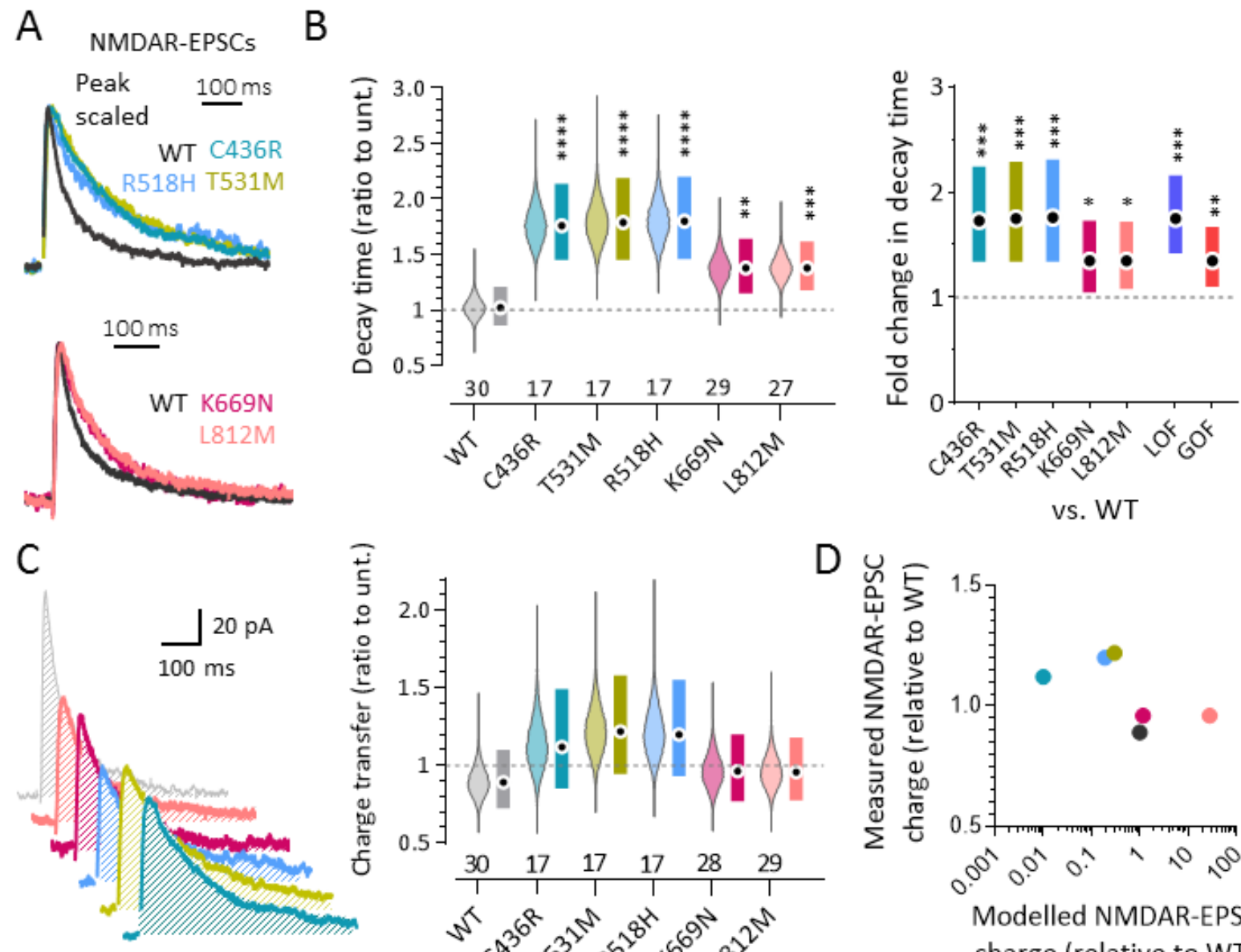

vs. WT
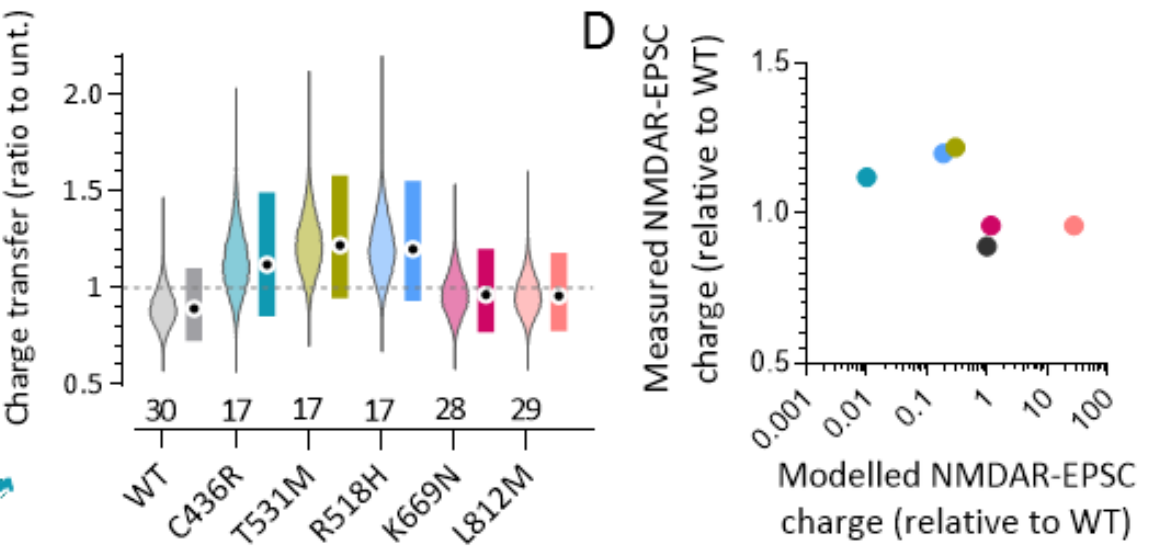

Figure 5: Gain- and loss-of-function LBD mutants are both associated with more prolonged NMDAR-mediated EPSCs

A. Peak-scaled, representative NMDAR-EPSCs traces from neurons rescued with LOF (top) or GOF (bottom) mutants are overlaid with WT to illustrate effects on the decay time course.

B. Violin plots of the posterior distributions along with their median and $95 \% \mathrm{Cls}$ for the transfected/untransfected ratios of the weighted decay time constant (left) and their contrasts versus WT control (right).

C. Left. Representative NMDAR-EPSCs for rescue with WT or mutants, shown with the area under the curve shaded to illustrate the calculation of charge transfer. Right. Violin plots of the posterior distributions along with their median and $95 \% \mathrm{Cls}$ for the transfected/untransfected ratios of the charge transferred during NMDAR-EPSCs. The upper limit of the y-axis is truncated at 2.2 D. Plot of measured versus modelled NMDAR-EPSC charge transfer. The method to model NMDAREPSC charge transfer is based on the approach of Swanger et al. (2016). 
A-D. NMDAR-EPSC conductances were calculated from current recordings at $+20 \mathrm{mV}$ by subtracting the AMPAR-EPSC conductance recorded at $-100 \mathrm{mV}$ (see methods).

B-D. Posterior distributions of the log-ratios were simulated using Bayesian multivariate multilevel modelling with a standard logistic distribution for a prior (see methods). The number of neuron pairs is provided at the base of each graph. 
Figure 6
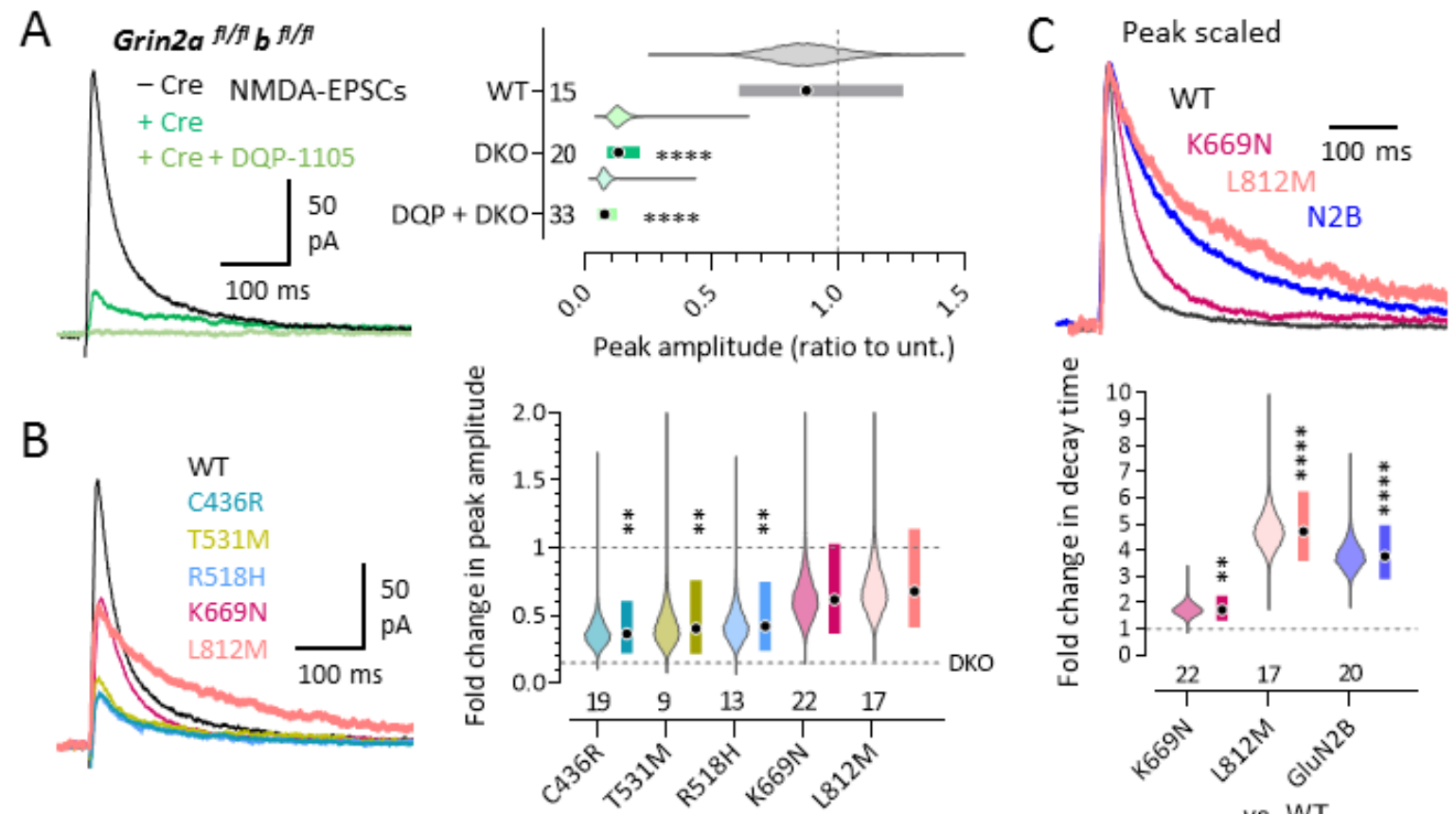

D

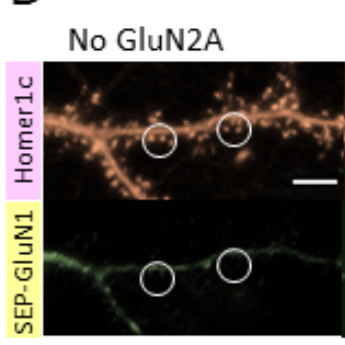

C436R
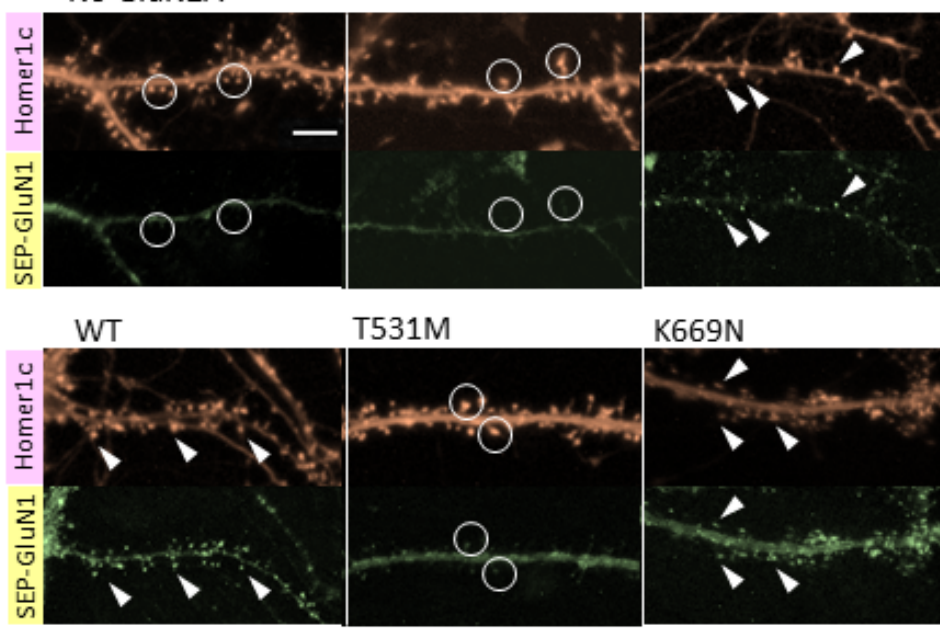

T531M

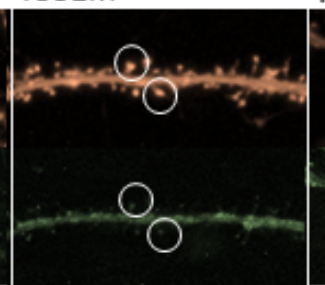

K669N

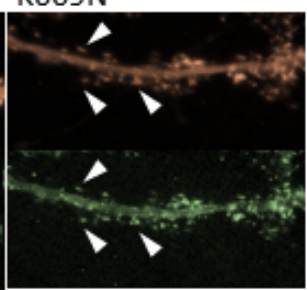

E

vs. WT
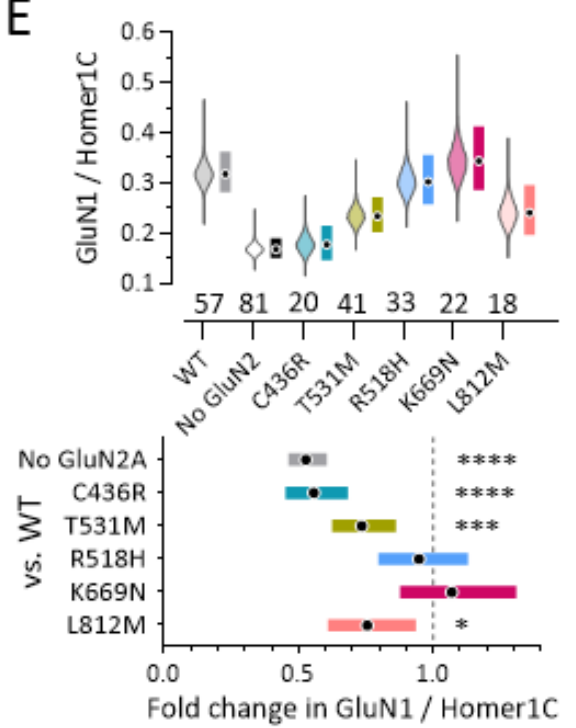

Figure 6: Contrasting mechanisms underlie synaptic dysfunction resulting from gainand loss-of-function GluN2A mutants

A. Left. Representative NMDAR-EPSCs from Grin $2 a^{+/+} b^{+/+}$(WT) or Grin $2 a^{\%} b^{\%}$ (double KO, or DKO) neurons. The residual current in the DKO was effectively blocked by a selective GluN2C/D antagonist DQP-1105 (10 $\mu \mathrm{M})$. Right. Violin plots of the posterior distributions along with their median and $95 \% \mathrm{Cls}$ for the transfected/untransfected ratios for the peak amplitude of NMDAREPSCS. The upper limit of the $\mathrm{x}$-axis is truncated at 1.5. 
B. Left. Representative NMDAR-EPSCs from DKO neurons rescued with WT or mutant human GluN2A. Right. Violin plots of the posterior distributions along with their median and $95 \% \mathrm{Cls}$ for the fold change in transfected/untransfected ratios for the peak amplitude of NMDAR-EPSCS compared to WT. The upper limit of the $y$-axis is truncated at 1.5.

C. Top. Peak-scaled, representative NMDAR-EPSCs from neurons rescued with WT (GluN2A or 2B) or GOF GluN2A mutants (L812M or K669N) are overlaid to illustrate effects on the decay time course. Bottom. Violin plots of the posterior distributions along with their median and $95 \% \mathrm{Cls}$ for the fold change in transfected/untransfected ratios for the weighted decay time constant of NMDAR-EPSCS compared to WT.

D. Representative images of cultured neurons overexpressing SEP-GluN1 (green), GluN2A and Homer1C-tdTomato (red). Homer1C was used as a synaptic marker. Circles highlight examples of synapses that exhibit low-to-moderate levels of SEP-GluN1. Arrow heads point to synapses that have high synaptic levels of SEP-GluN1.

E. Violin plots of the posterior distributions along with their median and $95 \% \mathrm{Cls}$ for the synaptic NMDAR levels as quantified by the GluN1/Homer1C ratio (top) and their contrasts versus WT control (bottom).

A-C, E. Posterior distributions of the log-ratios were simulated using Bayesian multivariate multilevel modelling with a standard logistic distribution for a prior (see methods). The number of neuron pairs is provided at the base of each graph. 
Figure 7

A

A AMPAR-EPSCS

$\sin$

Unt.

WT $\underset{20 \mathrm{~ms}}{2} 20 \mathrm{pA}$
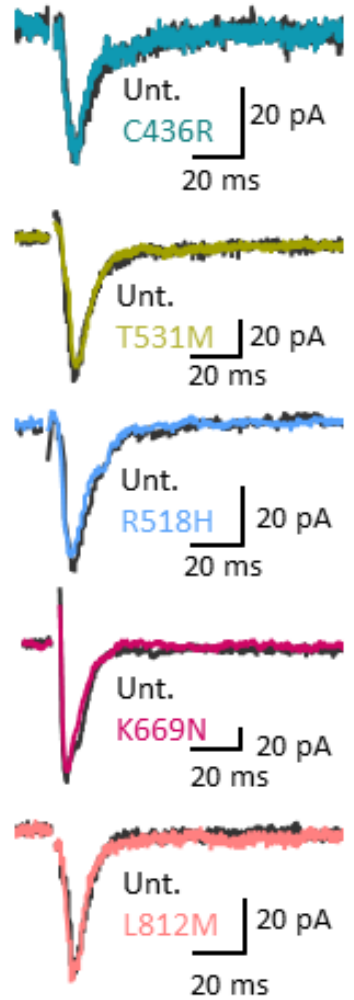

B

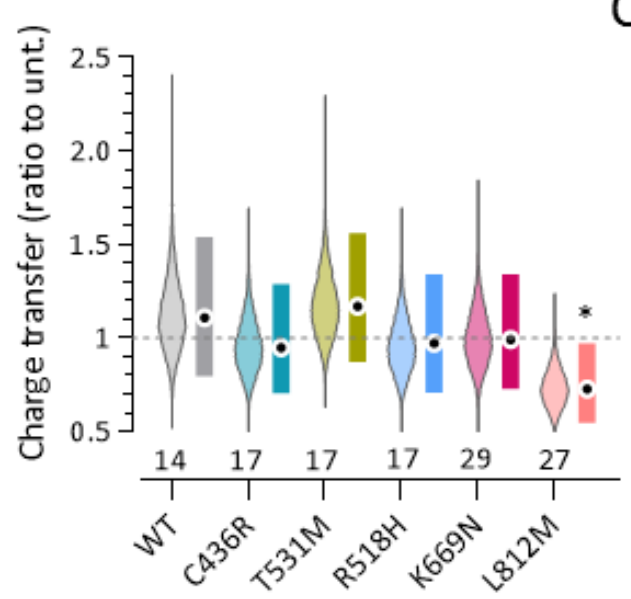

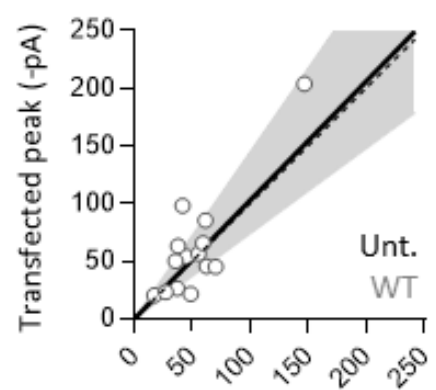
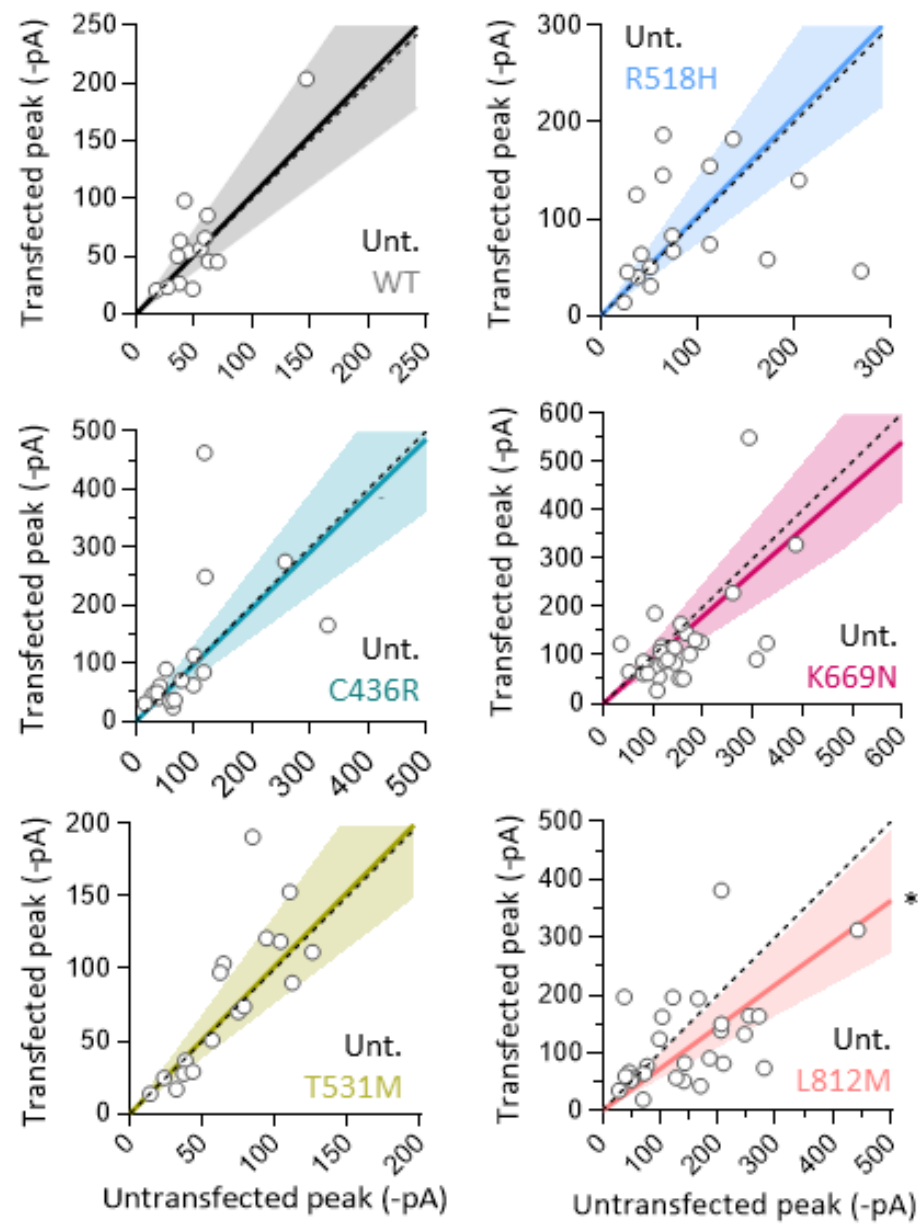

C

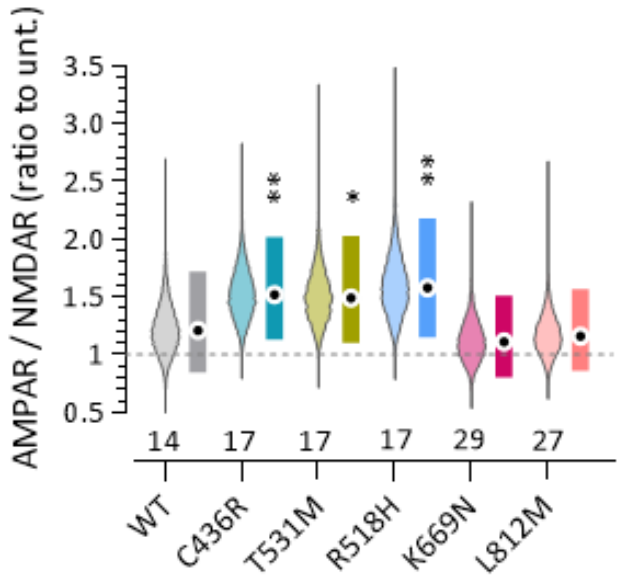




\section{Figure 7: GluN2A mutations are generally not associated with compensatory changes in AMPAR-EPSCs.}

A. Left: Representative traces of AMPAR-EPSCs from transfected and untransfected cell pairs. Transfected neurons represent rescue with human GluN2A (WT or mutant). Right: Scatter plots of NMDAR-EPSC peak amplitudes measured for pairs of transfected and untransfected neurons. Also graphed is the line of unity (dotted), median (solid) and $95 \% \mathrm{Cl}$ for the posterior distributions of the transfected/untransfected ratio (shaded).

B. Violin plots of the posterior distributions along with their median and $95 \% \mathrm{Cls}$ for the transfected/untransfected ratios of the charge transfer during AMPAR-EPSCs.

C. Violin plots of the posterior distributions along with their median and $95 \% \mathrm{Cls}$ for the transfected/untransfected ratios of the AMPAR/NMDAR ratio of EPSCs.

B-C. Posterior distributions of the log-ratios were simulated using Bayesian multivariate multilevel modelling with a standard logistic distribution for a prior (see methods). The number of neuron pairs is provided at the base of each graph. 


\section{Figure 8}
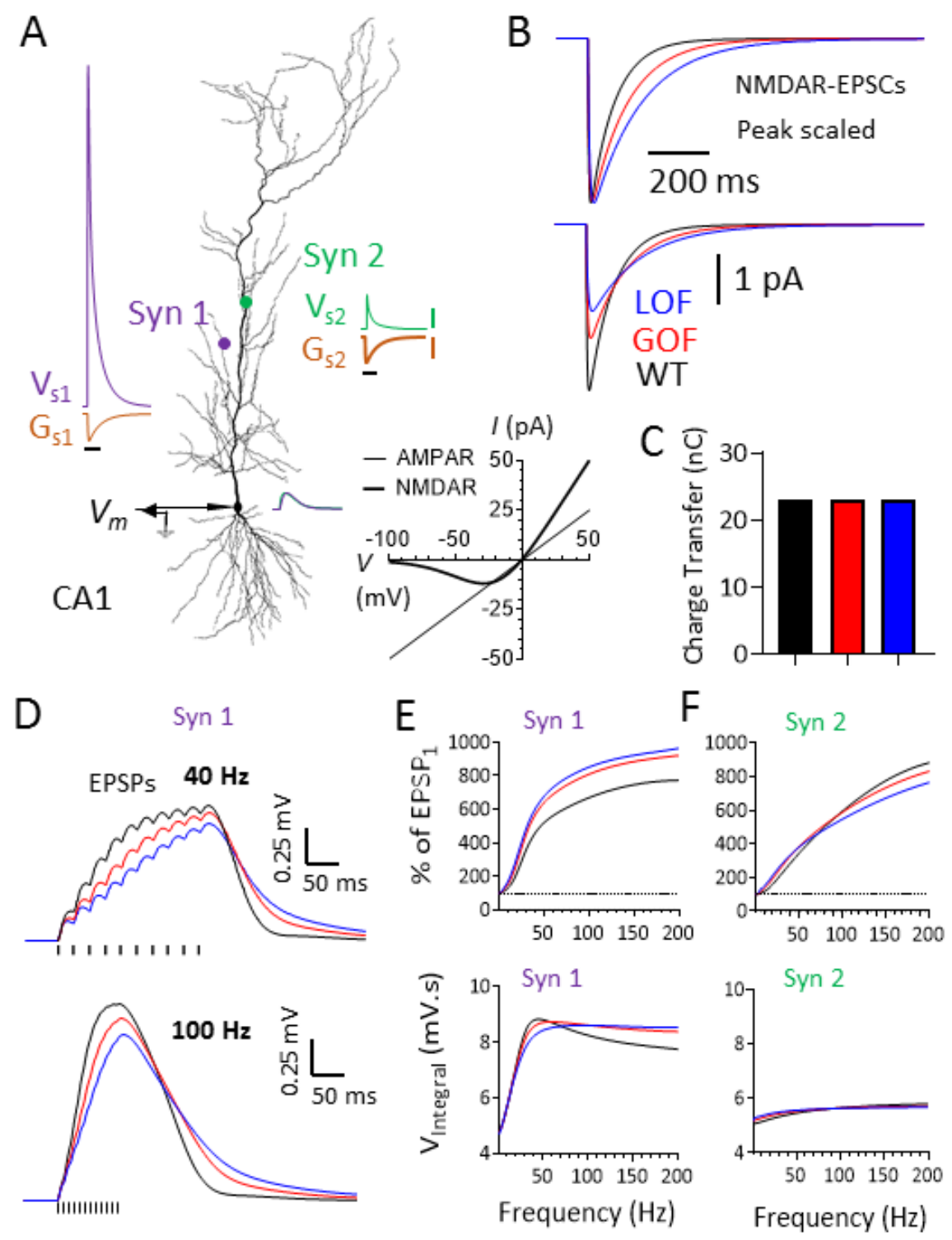

\section{Figure 8: Slower NMDAR-EPSC kinetics modify the temporal summation of EPSPs even when EPSC charge transfer is constant.}

A. Model of a CA1 neuron ${ }^{67}$. Identical synaptic conductances were introduced at synapse 1 (Syn1) and synapse 2 (Syn2). Syn1 was on an oblique dendrite while Syn2 was on the main apical branch. The local input resistance is higher at the Syn 1 than at Syn 2, resulting in a 10-fold larger local EPSP. Individual EPSPs generated by Syn1 and 2 are almost identical when measured at the soma. Inset. Current-voltage relationships of the AMPAR and NMDAR components of the synaptic conductances used at Syn 1 and 2. $V_{s 1}=$ membrane potential at Syn $1 ; G_{s 1}=$ Conductance at Syn $1 ; V_{s 2}=$ membrane potential at Syn 2; $\mathrm{G}_{\mathrm{s} 2}=$ Conductance at Syn 2; Vm = membrane potential at the soma; 
$\mathrm{R}_{\text {in }}=$ input resistance. Time base $=100 \mathrm{~ms}$; Conductance scale bars $(\mathrm{G}$, orange $)=0.4 \mathrm{nS}$; Membrane potential scale bars $(\mathrm{V}$, green and purple $)=0.2 \mathrm{mV}$.

B. Top. The kinetics of the NMDAR-component of the synaptic conductance was slowed to mimic the effects of GOF (red) and LOF (blue) mutants and this is illustrated with peak-scaled waveforms of the simulated NMDAR-EPSCs. Bottom. Example of simulated NMDAR-EPSCs above but with the amplitude $\left(g_{\max }\right)$ of the NMDAR-component reduced to maintain the overall charge transfer.

C. Bar graph illustrating the constant charge transfer during the EPSCs of varying kinetics to mimic GOF and LOF mutants.

D. Example $40 \mathrm{~Hz}$ (top) and $100 \mathrm{~Hz}$ (bottom) EPSP trains simulated for three different NMDAR conductances (WT, LOF and GOF) at Syn 1 and measured at the soma.

E. EPSP summation at Syn 1 as a function of train frequency. Top. Peak of the depolarization during the train as a percentage of the amplitude of a single EPSP is plotted as a function of train frequency. Bottom. The integral of the depolarization during the train is plotted as a function of train frequency.

F. As in E but for Syn 2. 


\section{Figure 9}

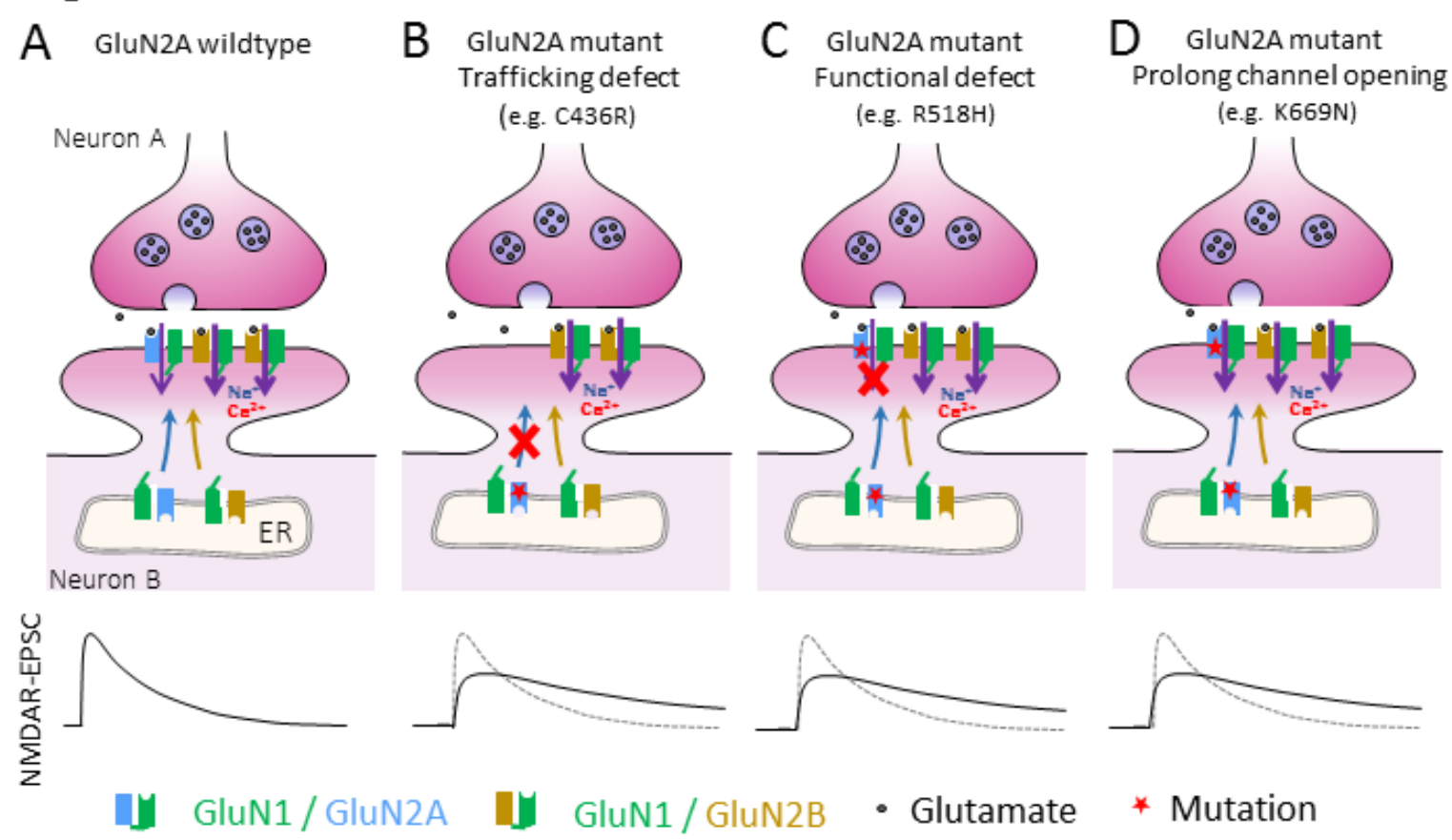

Figure 9: Model summarizing how different molecular defects can converge on similar NMDAR-mediated EPSCs
A. WT GluN2A and GluN2B contribute to NMDAR-EPSCs. GluN2B-containing NMDARs have more prolonged time course and cation influx as illustrated by the bold purple arrows.
B. GluN2A mutants with defects culminating in low levels of GluN2A at synapses result in NMDAR- EPSCs that are dominated by residual GluN2B-containing NMDARs.
C. GluN2A mutants that traffic to the synapse but are functionally defective result in NMDAR-EPSCS that are dominated by residual GluN2B-containing NMDARs.
D. GluN2A mutants that traffic to the synapse but have slower deactivation kinetics directly contribute to prolonging NMDAR-EPSCS.

B-D. The effect of mutations may result in a combination of these different mechanisms. 


\section{References}

1. Zito, K. \& Scheuss, V. NMDA Receptor Function and Physiological Modulation. in Encyclopedia of Neuroscience (ed. Squire, L. R.) 1157-1164 (Academic Press, 2009). doi:10.1016/B978008045046-9.01225-0.

2. Forrest, D. et al. Targeted disruption of NMDA receptor 1 gene abolishes NMDA response and results in neonatal death. Neuron 13, 325-338 (1994).

3. Hamada, S. et al. The glutamate receptor GluN2 subunit regulates synaptic trafficking of AMPA receptors in the neonatal mouse brain. Eur. J. Neurosci. 40, 3136-3146 (2014).

4. Kutsuwada, T. et al. Impairment of suckling response, trigeminal neuronal pattern formation, and hippocampal LTD in NMDA receptor epsilon 2 subunit mutant mice. Neuron 16, 333-344 (1996).

5. Lai, K.-O. \& Ip, N. Y. Structural plasticity of dendritic spines: The underlying mechanisms and its dysregulation in brain disorders. Biochim. Biophys. Acta BBA - Mol. Basis Dis. 1832, 2257-2263 (2013).

6. Antic, S. D., Zhou, W.-L., Moore, A. R., Short, S. M. \& Ikonomu, K. D. The decade of the dendritic NMDA spike. J. Neurosci. Res. 88, 2991-3001 (2010).

7. Hunt, D. L. \& Castillo, P. E. Synaptic plasticity of NMDA receptors: mechanisms and functional implications. Curr. Opin. Neurobiol. 22, 496-508 (2012).

8. Major, G., Larkum, M. E. \& Schiller, J. Active properties of neocortical pyramidal neuron dendrites. Annu. Rev. Neurosci. 36, 1-24 (2013).

9. Nicoll, R. A. A Brief History of Long-Term Potentiation. Neuron 93, 281-290 (2017).

10. Paoletti, P., Bellone, C. \& Zhou, Q. NMDA receptor subunit diversity: impact on receptor properties, synaptic plasticity and disease. Nat. Rev. Neurosci. 14, 383-400 (2013). 
11. Shipton, O. A. \& Paulsen, O. GluN2A and GluN2B subunit-containing NMDA receptors in hippocampal plasticity. Philos. Trans. R. Soc. Lond. B. Biol. Sci. 369, 20130163 (2014).

12. Morris, R. G. M. NMDA receptors and memory encoding. Neuropharmacology 74, 32-40 (2013).

13. Higley, M. J. \& Sabatini, B. L. Calcium Signaling in Dendritic Spines. Cold Spring Harb. Perspect. Biol. 4, a005686 (2012).

14. Cull-Candy, S., Brickley, S. \& Farrant, M. NMDA receptor subunits: diversity, development and disease. Curr. Opin. Neurobiol. 11, 327-335 (2001).

15. Laube, B., Hirai, H., Sturgess, M., Betz, H. \& Kuhse, J. Molecular determinants of agonist discrimination by NMDA receptor subunits: analysis of the glutamate binding site on the NR2B subunit. Neuron 18, 493-503 (1997).

16. Vicini, S. et al. Functional and Pharmacological Differences Between RecombinantN-Methyl-dAspartate Receptors. J. Neurophysiol. 79, 555-566 (1998).

17. Wyllie, D. J. A., Livesey, M. R. \& Hardingham, G. E. Influence of GluN2 subunit identity on NMDA receptor function. Neuropharmacology 74, 4-17 (2013).

18. Monyer, H., Burnashev, N., Laurie, D. J., Sakmann, B. \& Seeburg, P. H. Developmental and regional expression in the rat brain and functional properties of four NMDA receptors. Neuron $12,529-540$ (1994).

19. Scherzer, C. R. et al. Expression of N-Methyl-D-Aspartate receptor subunit mRNAs in the human brain: Hippocampus and cortex. J. Comp. Neurol. 390, 75-90 (1998).

20. Gray, J. A. et al. Distinct modes of AMPA receptor suppression at developing synapses by GluN2A and GluN2B: single-cell NMDA receptor subunit deletion in vivo. Neuron 71, 1085-1101 (2011).

21. Hestrin, S. Developmental regulation of NMDA receptor-mediated synaptic currents at a central synapse. Nature 357, 686-689 (1992). 
22. Liu, X.-B., Murray, K. D. \& Jones, E. G. Switching of NMDA receptor 2 A and 2B subunits at thalamic and cortical synapses during early postnatal development. J. Neurosci. Off. J. Soc. Neurosci. 24, 8885-8895 (2004).

23. Sheng, M., Cummings, J., Roldan, L. A., Jan, Y. N. \& Jan, L. Y. Changing subunit composition of heteromeric NMDA receptors during development of rat cortex. Nature 368, 144-147 (1994).

24. Barria, A. \& Malinow, R. Subunit-specific NMDA receptor trafficking to synapses. Neuron 35, 345-353 (2002).

25. Bellone, C. \& Nicoll, R. A. Rapid Bidirectional Switching of Synaptic NMDA Receptors. Neuron 55, 779-785 (2007).

26. Groc, L. et al. NMDA receptor surface mobility depends on NR2A-2B subunits. Proc. Natl. Acad. Sci. U. S. A. 103, 18769-18774 (2006).

27. Carmignoto, G. \& Vicini, S. Activity-dependent decrease in NMDA receptor responses during development of the visual cortex. Science 258, 1007-1011 (1992).

28. Philpot, B. D., Sekhar, A. K., Shouval, H. Z. \& Bear, M. F. Visual experience and deprivation bidirectionally modify the composition and function of NMDA receptors in visual cortex. Neuron 29, 157-169 (2001).

29. Quinlan, E. M., Philpot, B. D., Huganir, R. L. \& Bear, M. F. Rapid, experience-dependent expression of synaptic NMDA receptors in visual cortex in vivo. Nat. Neurosci. 2, 352-357 (1999).

30. Roberts, E. B. \& Ramoa, A. S. Enhanced NR2A Subunit Expression and Decreased NMDA Receptor Decay Time at the Onset of Ocular Dominance Plasticity in the Ferret. J. Neurophysiol. 81, 2587-2591 (1999).

31. Sakimura, K. et al. Reduced hippocampal LTP and spatial learning in mice lacking NMDA receptor epsilon 1 subunit. Nature 373, 151-155 (1995). 
32. Salmi, M. et al. Impaired vocal communication, sleep-related discharges, and transient alteration of slow-wave sleep in developing mice lacking the GluN2A subunit of N-methyl-daspartate receptors. Epilepsia 60, 1424-1437 (2019).

33. Salmi, M. et al. Transient microstructural brain anomalies and epileptiform discharges in mice defective for epilepsy and language-related NMDA receptor subunit gene Grin2a. Epilepsia 59, 1919-1930 (2018).

34. Kiyama, Y. et al. Increased thresholds for long-term potentiation and contextual learning in mice lacking the NMDA-type glutamate receptor epsilon1 subunit. J. Neurosci. 18, 6704-6712 (1998).

35. Myers, K. A., Johnstone, D. L. \& Dyment, D. A. Epilepsy genetics: Current knowledge, applications, and future directions. Clin. Genet. 95, 95-111 (2019).

36. Endele, S. et al. Mutations in GRIN2A and GRIN2B encoding regulatory subunits of NMDA receptors cause variable neurodevelopmental phenotypes. Nat. Genet. 42, 1021-1026 (2010).

37. Firth, H. V. et al. DECIPHER: Database of Chromosomal Imbalance and Phenotype in Humans Using Ensembl Resources. Am. J. Hum. Genet. 84, 524-533 (2009).

38. Reutlinger, C. et al. Deletions in $16 \mathrm{p} 13$ including GRIN2A in patients with intellectual disability, various dysmorphic features, and seizure disorders of the rolandic region. Epilepsia 51, 18701873 (2010).

39. Myers, K. A. \& Scheffer, I. E. GRIN2A-Related Speech Disorders and Epilepsy. in GeneReviews ${ }^{\circledR}$ (eds. Adam, M. P. et al.) (University of Washington, Seattle, 2016).

40. Strehlow, V. et al. GRIN2A-related disorders: genotype and functional consequence predict phenotype. Brain 142, 80-92 (2019).

41. XiangWei, W., Jiang, Y. \& Yuan, H. De novo mutations and rare variants occurring in NMDA receptors. Curr. Opin. Physiol. 2, 27-35 (2018). 
42. Carvill, G. L. et al. GRIN2A mutations cause epilepsy-aphasia spectrum disorders. Nat. Genet.

45, 1073-1076 (2013).

43. Lemke, J. R. et al. Mutations in GRIN2A cause idiopathic focal epilepsy with rolandic spikes. Nat.

Genet. 45, 1067-1072 (2013).

44. Lesca, G. et al. GRIN2A mutations in acquired epileptic aphasia and related childhood focal epilepsies and encephalopathies with speech and language dysfunction. Nat. Genet. 45, 10611066 (2013).

45. Turner, S. J., Morgan, A. T., Perez, E. R. \& Scheffer, I. E. New genes for focal epilepsies with speech and language disorders. Curr. Neurol. Neurosci. Rep. 15, 35 (2015).

46. Yang, X. et al. GRIN2A mutations in epilepsy-aphasia spectrum disorders. Brain Dev. 40, 205210 (2018).

47. Gobbi, G., Boni, A. \& Filippini, M. The spectrum of idiopathic Rolandic epilepsy syndromes and idiopathic occipital epilepsies: from the benign to the disabling. Epilepsia $\mathbf{4 7}$ Suppl 2, 62-66 (2006).

48. Addis, L. et al. Epilepsy-associated GRIN2A mutations reduce NMDA receptor trafficking and agonist potency - molecular profiling and functional rescue. Sci. Rep. 7, 66 (2017).

49. CFERV. Center for Functional Evaluation of Rare variants.

http://functionalvariants.emory.edu/index.html (2017).

50. Chen, W. et al. Functional Evaluation of a De Novo GRIN2A Mutation Identified in a Patient with Profound Global Developmental Delay and Refractory Epilepsy. Mol. Pharmacol. 91, 317-330 (2017).

51. Gao, K. et al. A de novo loss-of-function GRIN2A mutation associated with childhood focal epilepsy and acquired epileptic aphasia. PloS One 12, e0170818 (2017). 
52. Ogden, K. K. et al. Molecular Mechanism of Disease-Associated Mutations in the Pre-M1 Helix of NMDA Receptors and Potential Rescue Pharmacology. PLoS Genet. 13, e1006536 (2017).

53. Sibarov, D. A. et al. Functional Properties of Human NMDA Receptors Associated with EpilepsyRelated Mutations of GluN2A Subunit. Front. Cell. Neurosci. 11, 155 (2017).

54. Swanger, S. A. et al. Mechanistic Insight into NMDA Receptor Dysregulation by Rare Variants in the GluN2A and GluN2B Agonist Binding Domains. Am. J. Hum. Genet. 99, 1261-1280 (2016).

55. Yuan, H. et al. Functional analysis of a de novo GRIN2A missense mutation associated with early-onset epileptic encephalopathy. Nat. Commun. 5, 3251 (2014).

56. Serraz, B., Grand, T. \& Paoletti, P. Altered zinc sensitivity of NMDA receptors harboring clinically-relevant mutations. Neuropharmacology 109, 196-204 (2016).

57. Sceniak, M. P. et al. A GluN2B mutation identified in Autism prevents NMDA receptor trafficking and interferes with dendrite growth. J. Cell Sci. (2019) doi:10.1242/jcs.232892.

58. Schorge, S. \& Colquhoun, D. Studies of NMDA Receptor Function and Stoichiometry with Truncated and Tandem Subunits. J. Neurosci. 23, 1151-1158 (2003).

59. Kurosaki, T., Popp, M. W. \& Maquat, L. E. Quality and quantity control of gene expression by nonsense-mediated mRNA decay. Nat. Rev. Mol. Cell Biol. 20, 406-420 (2019).

60. Long, A. A. et al. The nonsense-mediated decay pathway maintains synapse architecture and synaptic vesicle cycle efficacy. J. Cell Sci. 123, 3303-3315 (2010).

61. Nagy, A. Cre recombinase: The universal reagent for genome tailoring. genesis 26, 99-109 (2000).

62. Madry, C., Mesic, I., Betz, H. \& Laube, B. The N-terminal domains of both NR1 and NR2 subunits determine allosteric $\mathrm{Zn} 2+$ inhibition and glycine affinity of $\mathrm{N}$-methyl-D-aspartate receptors. Mol. Pharmacol. 72, 1535-1544 (2007). 
63. Puddifoot, C. A., Chen, P. E., Schoepfer, R. \& Wyllie, D. J. A. Pharmacological characterization of recombinant NR1/NR2A NMDA receptors with truncated and deleted carboxy termini expressed in Xenopus laevis oocytes. Br. J. Pharmacol. 156, 509-518 (2009).

64. Horak, M., Petralia, R. S., Kaniakova, M. \& Sans, N. ER to synapse trafficking of NMDA receptors. Front. Cell. Neurosci. 8, 394 (2014).

65. Kopec, C. D., Li, B., Wei, W., Boehm, J. \& Malinow, R. Glutamate receptor exocytosis and spine enlargement during chemically induced long-term potentiation. J. Neurosci. 26, 2000-2009 (2006).

66. Adesnik, H., Li, G., During, M. J., Pleasure, S. J. \& Nicoll, R. A. NMDA receptors inhibit synapse unsilencing during brain development. Proc. Natl. Acad. Sci. U. S. A. 105, 5597-5602 (2008).

67. Golding, N. L., Mickus, T. J., Katz, Y., Kath, W. L. \& Spruston, N. Factors mediating powerful voltage attenuation along CA1 pyramidal neuron dendrites. J. Physiol. 568, 69-82 (2005).

68. Moradi, K. et al. A fast model of voltage-dependent NMDA receptors. J. Comput. Neurosci. 34, 521-531 (2013).

69. Stroebel, D., Casado, M. \& Paoletti, P. Triheteromeric NMDA receptors: from structure to synaptic physiology. Curr. Opin. Physiol. 2, 1-12 (2018).

70. Kellermayer, B. et al. Differential Nanoscale Topography and Functional Role of GluN2-NMDA Receptor Subtypes at Glutamatergic Synapses. Neuron 100, 106-119.e7 (2018).

71. Akashi, K. et al. NMDA receptor GluN2B (GluR epsilon 2/NR2B) subunit is crucial for channel function, postsynaptic macromolecular organization, and actin cytoskeleton at hippocampal CA3 synapses. J. Neurosci. Off. J. Soc. Neurosci. 29, 10869-10882 (2009).

72. Rathenberg, J., Nevian, T. \& Witzemann, V. High-efficiency transfection of individual neurons using modified electrophysiology techniques. J. Neurosci. Methods 126, 91-98 (2003). 
73. Campagnola, L., Kratz, M. B. \& Manis, P. B. ACQ4: an open-source software platform for data acquisition and analysis in neurophysiology research. Front. Neuroinformatics 8, 3 (2014).

74. Jiang, M. \& Chen, G. High Ca2+-phosphate transfection efficiency in low-density neuronal cultures. Nat. Protoc. 1, 695-700 (2006).

75. Guzman, S. J., Schlögl, A. \& Schmidt-Hieber, C. Stimfit: quantifying electrophysiological data with Python. Front. Neuroinformatics 8, 16 (2014).

76. Schindelin, J. et al. Fiji: an open-source platform for biological-image analysis. Nat. Methods $\mathbf{9}$, $676-682(2012)$.

77. Rueden, C. T. et al. ImageJ2: ImageJ for the next generation of scientific image data. BMC Bioinformatics 18, 529 (2017).

78. Carnevale, N. T. \& Hines, M. L. The NEURON Book. (Cambridge University Press, 2006).

79. Hubert, M., Rousseeuw, P. J. \& Branden, K. V. ROBPCA: A New Approach to Robust Principal Component Analysis: Technometrics: Vol 47, No 1. (2005).

80. Verboven, S. \& Hubert, M. LIBRA: a MATLAB library for robust analysis. Chemom. Intell. Lab. Syst. 75, 127-136 (2005).

81. Bürkner, P.-C. brms: An R Package for Bayesian Multilevel Models Using Stan. J. Stat. Softw. 80, $1-28$ (2017).

82. Makowski, D., Ben-Shachar, M. S., Chen, S. H. A. \& Lüdecke, D. Indices of Effect Existence and Significance in the Bayesian Framework. Front. Psychol. 10, (2019).

83. Gelman, A., Hill, J. \& Yajima, M. Why We (Usually) Don't Have to Worry About Multiple Comparisons. J. Res. Educ. Eff. 5, 189-211 (2012). 